\title{
Quantum Anomalous Hall Effects in Graphene from Proximity-Induced Uniform and Staggered Spin-Orbit and Exchange Coupling
}

\author{
Petra Högl, ${ }^{1, \text { | }}$ Tobias Frank, ${ }^{1}$ Klaus Zollner, ${ }^{1}$ Denis Kochan, ${ }^{1}$ Martin Gmitra, ${ }^{2}$ and Jaroslav Fabian ${ }^{1}$ \\ ${ }^{1}$ Institute for Theoretical Physics, University of Regensburg, 93040 Regensburg, Germany \\ ${ }^{2}$ Department of Theoretical Physics and Astrophysics, \\ Pavol Jozef Šafárik University, 04001 Košice, Slovakia
}

\begin{abstract}
We investigate an effective model of proximity modified graphene (or symmetrylike materials) with broken time-reversal symmetry. We predict the appearance of quantum anomalous Hall phases by computing bulk band gap and Chern numbers for benchmark combinations of system parameters. Allowing for staggered exchange field enables quantum anomalous Hall effect in flat graphene with Chern number $C=1$. We explicitly show edge states in zigzag and armchair nanoribbons and explore their localization behavior. Remarkably, the combination of staggered intrinsic spin-orbit and uniform exchange coupling gives topologically protected (unlike in time-reversal systems) pseudohelical states, whose spin is opposite in opposite zigzag edges. Rotating the magnetization from out of plane to in plane makes the system trivial, allowing to control topological phase transitions. We also propose, using density functional theory, a material platform - graphene on Ising antiferromagnet $\mathrm{MnPSe}_{3}$ - to realize staggered exchange (pseudospin Zeeman) coupling.
\end{abstract}

Topological effects in graphene 1 attract immense attention due to their fascinating physics and potential applications in dissipationless electronics and spintronics [2, 3]. In the quantum spin Hall effect topological edge states are protected by time-reversal symmetry [48, while the presence of an exchange coupling in the quantum anomalous Hall effect (QAHE) breaks timereversal symmetry, inducing topological protection described by Chern numbers [7, 9 14.

There has been enormous experimental progress towards realizing topological phases in graphene, by means of van der Waals heterostructures [15. In pristine graphene intrinsic spin-orbit coupling (SOC) is predicted to be weak, $12 \mu \mathrm{eV}$ [16]; a recent experiment for graphene on $\mathrm{SiO}_{2}$ found it to be $20 \mu \mathrm{eV}$ [17]. However, graphene on transition-metal dichalcogenides (TMDCs), such as $\mathrm{MoS}_{2}$ or $\mathrm{WSe}_{2}$, exhibits proximity SOC on meV scale 1829. But not only the magnitude of SOC becomes giant (compared to pristine graphene), the functional form of SOC changes as well. Instead of intrinsic SOC, graphene on TMDCs acquires staggered (valley-Zeeman) SOC with opposite sign on $A$ and $B$ sublattices, as spectacularly confirmed by spin relaxation anisotropy experiments 30 32. Staggered SOC leads to protected pseudohelical edge states [8] in the absence of magnetic interactions.

Adding magnetic exchange breaks time-reversal symmetry and together with SOC leads to QAHE. What if exchange coupling were also staggered, realizing proximity antiferromagnetic graphene? Thus far only uniform (ferromagnetic) exchange coupling was considered in the proximity effect of graphene $33-44$. Fortunately, there are now suitable candidates, layered semiconducting Ising antiferromagnets, which could serve as substrate for graphene and induce staggered exchange. One example is discussed below.

Our main goal is to make specific predictions for topo-

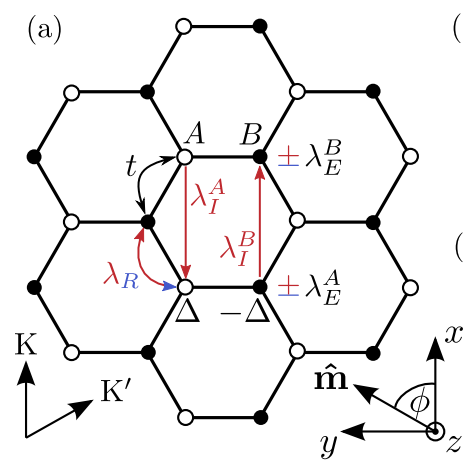

(b)

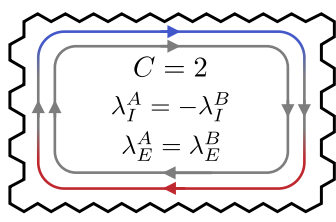

(c)

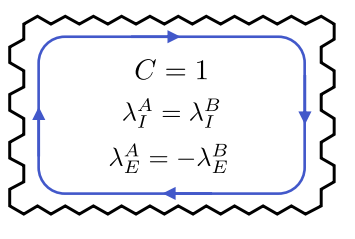

FIG. 1. (a) Scheme of graphene lattice with proximity induced hoppings. Sublattices $A$ and $B$ are denoted by empty and full dots, respectively. Color indicates action on spin. The minimal model contains spin neutral nearest-neighbor hopping $t$ and on site staggered potential $\Delta$; spin-mixing nearestneighbor Rashba SOC $\lambda_{R}$; spin and sublattice resolved next nearest-neighbor intrinsic SOC $\lambda_{I}^{A}, \lambda_{I}^{B}$; on site sublattice resolved exchange splitting $\lambda_{E}^{A}, \lambda_{E}^{B}$ (spin-dependent energy shift spin up + , spin down - ). Orientation of reciprocal lattice is shown by $K$ and $K^{\prime}$. Magnetization orientation in real space is specified by $\hat{\mathbf{m}}$. Sketch of quantum anomalous Hall states along zigzag and armchair edges for (b) $\lambda_{I}^{A}=-\lambda_{I}^{B}$ and $\lambda_{E}^{A}=\lambda_{E}^{B}$ and (c) $\lambda_{I}^{A}=\lambda_{I}^{B}$ and $\lambda_{E}^{A}=-\lambda_{E}^{B}$. The Chern number is given by $C$. Color indicates $\hat{s}_{z}$ spin expectation value: red spin up, blue spin down, gray unpolarized.

logical phases in graphene (or symmetrylike materials) by considering those newly unveiled staggered regimes for spin-orbit and exchange couplings. In addition to QAHE phases with Chern number $C=2$ (as in KaneMele models for uniform couplings), we find $C=1$ (single edge state) for uniform SOC and staggered exchange. Remarkably, rotating the exchange to the plane makes the system topologically trivial, making the magnetoanisotropy an effective knob on the topological prop- 
erties. To support our model calculations we also introduce a platform for making graphene a proximity antiferromagnet. Using density functional theory, we calculate the electronic states of graphene on monolayer $\mathrm{MnPSe}_{3}$, which is an Ising-type antiferromagnetic semiconductor. Graphene's Dirac states are well preserved displaying clear signatures of staggered (pseudospin Zeeman) exchange, also confirmed by tight-binding fitting.

Model. We consider graphene modified by a proximity effect such that the sublattice, horizontal reflection, and time-reversal symmetries are broken. The minimal $C_{3 v}$-symmetric Hamiltonian can be written as

$$
\begin{aligned}
\mathcal{H}= & -t \sum_{\langle i, j\rangle, \sigma} c_{i \sigma}^{\dagger} c_{j \sigma}+\Delta \sum_{i, \sigma} \xi_{i} c_{i \sigma}^{\dagger} c_{i \sigma} \\
& +\frac{2 i \lambda_{R}}{3} \sum_{\langle i, j\rangle, \sigma, \sigma^{\prime}} c_{i \sigma}^{\dagger} c_{j \sigma^{\prime}}\left[\left(\hat{\mathbf{s}} \times \mathbf{d}_{i j}\right)_{z}\right]_{\sigma \sigma^{\prime}} \\
& +\frac{i}{3 \sqrt{3}} \sum_{\langle\langle i, j\rangle\rangle, \sigma, \sigma^{\prime}} \lambda_{I}^{i} \nu_{i j} c_{i \sigma}^{\dagger} c_{j \sigma^{\prime}}\left[\hat{\mathbf{s}}_{z}\right]_{\sigma \sigma^{\prime}} \\
& +\sum_{i, \sigma, \sigma^{\prime}} \lambda_{E}^{i} c_{i \sigma}^{\dagger} c_{i \sigma^{\prime}}[\hat{\mathbf{m}} \cdot \hat{\mathbf{s}}]_{\sigma \sigma^{\prime}},
\end{aligned}
$$

where $c_{i \sigma}^{\dagger}\left(c_{i \sigma}\right)$ is the creation (annihilation) operator for an electron on lattice site $i$ that belongs to the sublattice $A$ or $B$ and carries spin $\sigma$. The hoppings are depicted in Fig. 1(a). The first two terms are the spin-preserving nearest-neighbor hopping (sum over $\langle i, j\rangle$ ) and the staggered on site potential $\Delta$ with $\xi_{i}=1$ on sublattice $A$ and $\xi_{i}=-1$ on $B$. The potential difference on $A$ and $B$ takes into account the different environment that atoms in sublattices $A$ and $B$ encounter in a heterostructure (implicitly broken sublattice symmetry). The next two terms describe SOC 45. Rashba SOC $\lambda_{R}$ mixes spins of nearest neighbors, where the unit vector $\mathbf{d}_{i j}$ points from site $j$ to $i$ and $\hat{\mathbf{s}}$ contains spin Pauli matrices. It occurs when inversion symmetry is broken, e.g., in an asymmetric heterostructure. Intrinsic SOC couples the same spins on next-nearest neighbors (sum over $\langle\langle i, j\rangle\rangle$ ). It depends on clockwise $\left(\nu_{i j}=-1\right)$ or counterclockwise $\left(\nu_{i j}=1\right)$ hopping paths from site $j$ to $i$ and is sublattice resolved, $\lambda_{I}^{i}$ with $i=A, B$. The above described terms form an experimentally relevant model for nonmagnetic graphene proximity systems 8 , 19, 45, 46 extending the earlier models from McClure and Yafet [47, Haldane [4, and Kane and Mele [5]. The last term in Eq. (1) extends the model to magnetic systems. It is a sublatticedependent exchange coupling that introduces spin magnetization and breaks time-reversal symmetry [33. Similar to the intrinsic SOC we allow for different values $\lambda_{E}^{i}$ with $i=A, B$ and orientation of magnetization along the unit vector $\hat{\mathbf{m}}=(\cos \phi \sin \theta, \sin \phi \sin \theta, \cos \theta)$, where $\phi$ is measured with respect to the $x$-axis and $\theta$ with respect to the $z$-axis in Fig. 1(a).

The Hamiltonian in Eq. (1) breaks time-reversal, particle-hole, and chiral symmetry. Therefore it belongs
TABLE I. Labeling and corresponding Chern numbers for the four combinations of (out-of-plane) exchange $(E)$ and intrinsic $(I)$ spin-orbit couplings on $A$ and $B$ atoms, summarizing Fig. 2 ,

\begin{tabular}{l|c|c|c}
\hline \hline \multicolumn{2}{c|}{ couplings } & $\lambda_{E}^{A}=\lambda_{E}^{B}$ & $\lambda_{E}^{A}=-\lambda_{E}^{B}$ \\
\hline \multirow{2}{*}{$\lambda_{I}^{A}=\lambda_{I}^{B}$} & label & $(\mathrm{uu})$ & $(\mathrm{us})$ \\
& Chern & $0, \pm 2$ & $0, \pm 1$ \\
\hline \multirow{2}{*}{$\lambda_{I}^{A}=-\lambda_{I}^{B}$} & label & $(\mathrm{su})$ & $(\mathrm{ss})$ \\
& Chern & $0, \pm 2$ & 0 \\
\hline \hline
\end{tabular}

to class $\mathrm{A}$ of $2 \mathrm{D}$ quantum Hall systems and its topological nature can be determined by the Chern number $C$ [48, 49]. A nonzero $C$ indicates a topological system, namely, a QAHE phase. As for the quantum Hall state the Chern number gives the quantized Hall conductance $\sigma_{H}=C e^{2} / h$ [48. Every change of the Chern number is accompanied by the closing of the bulk band gap.

Since we wish to present material-independent topological phase diagrams, we fix the model parameters to generic values $\Delta=0.1 t$ and $\lambda_{R}=(3 / 2) \times 0.05 t$, and explore the interplay of intrinsic SOC and exchange coupling first for the out-of-plane magnetization $\hat{\mathbf{m}}=$ $(0,0,1)$. For the intrinsic SOC we consider two possibilities: a uniform (u) intrinsic coupling, $\lambda_{I}^{A}=\lambda_{I}^{B}$, which is of the conventional McClure-Yafet-Kane-Mele type 5 , 47, recently predicted to be giant $(0.5 \mathrm{eV})$ in the monolayer jacutingaite [50; a staggered (s) intrinsic SOC $\lambda_{I}^{A}=-\lambda_{I}^{B}$, which was predicted [18 20] and experimentally confirmed 29, 30, 32 for graphene on TMDCs. This (also called valley-Zeeman) coupling seems not to be restricted to valley-Zeeman substrates - it is also predicted to come from topological insulator $\mathrm{Bi}_{2} \mathrm{Se}_{3}$ [51. Similarly, we consider a uniform exchange coupling, $\lambda_{E}^{A}=\lambda_{E}^{B}$, which would come from graphene on an Ising ferromagnet, and a staggered exchange, $\lambda_{E}^{A}=-\lambda_{E}^{B}$, which could be realized by placing graphene on an Ising antiferromagnet (see below). Overall we have four possible combinations: uniform-uniform (uu) $\lambda_{I}^{A}=\lambda_{I}^{B}$ and $\lambda_{E}^{A}=$ $\lambda_{E}^{B}$, uniform-staggered (us) $\lambda_{I}^{A}=\lambda_{I}^{B}$ and $\lambda_{E}^{A}=-\lambda_{E}^{B}$, staggered-uniform (su) $\lambda_{I}^{A}=-\lambda_{I}^{B}$ and $\lambda_{E}^{A}=\lambda_{E}^{B}$, and staggered-staggered (ss) $\lambda_{I}^{A}=-\lambda_{I}^{B}$ and $\lambda_{E}^{A}=-\lambda_{E}^{B}$; see Table 【

QAHE phases. In Fig. 2 we show the bulk band gap and Chern number. We find the following for the studied case: (su) two magnetic Chern insulator phases with $C= \pm 2$; (us) four nontrivial regions with $C= \pm 1$; (uu) two QAHE phases with $C= \pm 2$; (ss) trivial insulating or metallic system. Analytical conditions to be in a nontrivial phase are given in [52. Case (uu) is an extension of Ref. 9 including both staggered potential and intrinsic SOC. Cases (su) and (uu) require finite Rashba SOC to have a gapped system in the vicinity of the Dirac points. 
For uniform SOC but staggered exchange (us), no Rashba coupling is needed to induce QAHE. This case allows for single propagating states, which would be a signature for the antiferromagnetic QAHE under the assumption that the absolute value of the parameters of the $A$ and $B$ sublattices do not differ drastically. The discussed topological phases also exist when the exact uniform or staggered condition is relaxed as shown in the Supplemental Material [52. The Chern numbers are summarized in Table I. Some aspects of the (us) and (ss) physics, without staggered $\Delta$, was very recently discussed in Ref. [53].

Proposal for proximity antiferromagnetic graphene. We now present a specific proposal for making graphene antiferromagnetic and introduce a material platform for cases (us) and (ss), or simply staggered exchange. The platform is graphene on monolayer $\mathrm{MnPSe}_{3}$, which is an Ising antiferromagnet and semiconductor [54, 55]. Since bulk $\mathrm{MnPSe}_{3}$ is a layered compound (with optical gap $2.3 \mathrm{eV} 56$ ), and only the top monolayer is important for proximity effects, the platform can be experimentally realized using a $\mathrm{MnPSe}_{3}$ film. There is an earlier proposal [57] to place graphene on an antiferromagnet, perovskite $\mathrm{BiFeO}_{3}$, but the $\mathrm{Fe}$ (111) plane that proximitizes

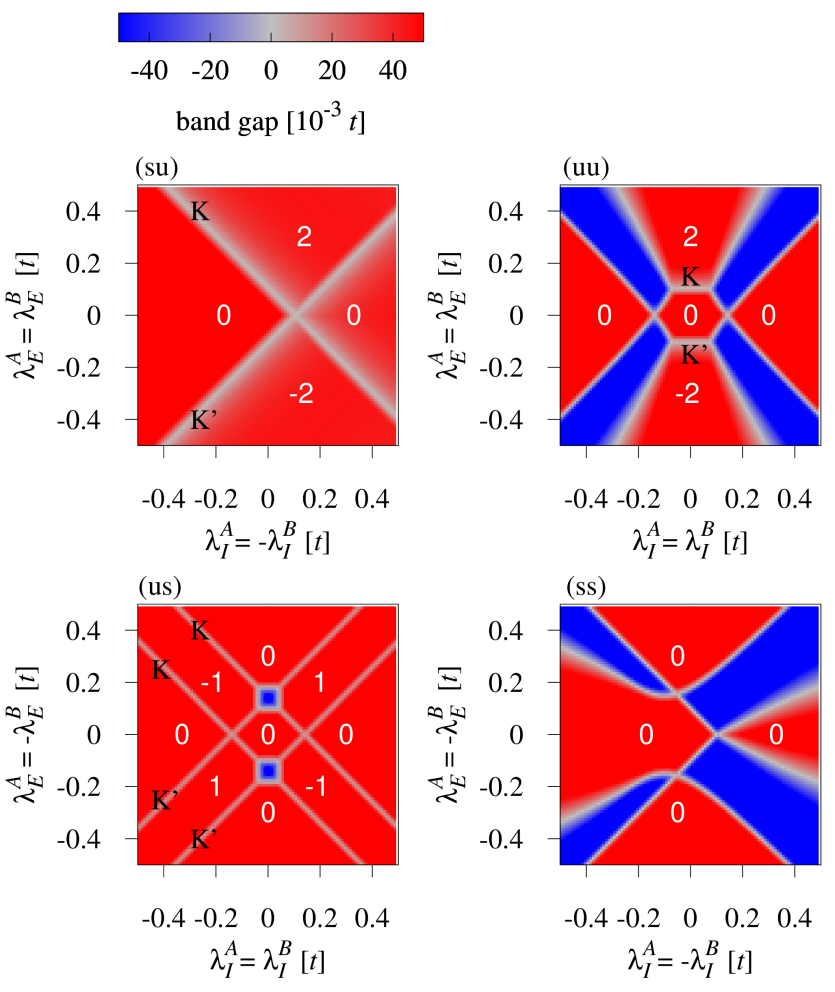

FIG. 2. Topological phases of magnetic graphene with SOC. Global bulk band gap and Chern number (white numbers) for $\Delta=0.1 t$ and $\lambda_{R}=(3 / 2) \times 0.05 t$ varying intrinsic SOC $\lambda_{I}^{A}, \lambda_{I}^{B}$ and exchange splitting $\lambda_{E}^{A}, \lambda_{E}^{B}$ for out-of-plane magnetization $\hat{\mathbf{m}}=(0,0,1)$. Position of gap closing in $\mathbf{k}$-space is specified by K, K'. Negative band gap indicates transition to metallic system due to indirect band gap closing from bands at different $\mathbf{k}$ values. graphene is ferromagnetic, inducing a ferromagnetic (as also in [11, 13]) and not antiferromagnetic exchange in graphene.

In Figs. 3(a)-3 (3) we show the investigated atomic and calculated electronic structure of graphene on monolayer $\mathrm{MnPSe}_{3}$ where Mn forms a hexagonal lattice with alternating out-of-plane magnetization. Details of the calculations are described in the Supplemental Material [52]. The Dirac states from graphene are nicely preserved and reside within the band gap of $\mathrm{MnPSe}_{3}$. An enlargement of the low energy bands around the $\mathrm{K}$ point reveals proximity-induced staggered exchange splitting of the bands. Our model Hamiltonian [52] fits the low energy dispersion and spin splittings, see Figs. $3(\mathrm{~d}, \mathrm{e})$.

For our heterostructure, the splitting between spin up and spin down bands $\mathrm{E}_{\mathrm{up}}-\mathrm{E}_{\mathrm{dn}}$ for the valence band (VB) is negative, while the one for the conduction band (CB) is positive at the $\mathrm{K}$ point, see Fig. 3(e). Even though the antiferromagnetic hexagonal Mn-lattice is not commensurate with the graphene lattice, we effectively get different spin splittings for VB and CB. The fitting parameters (a)

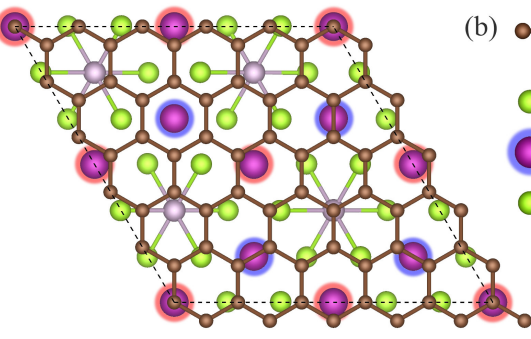

(b)
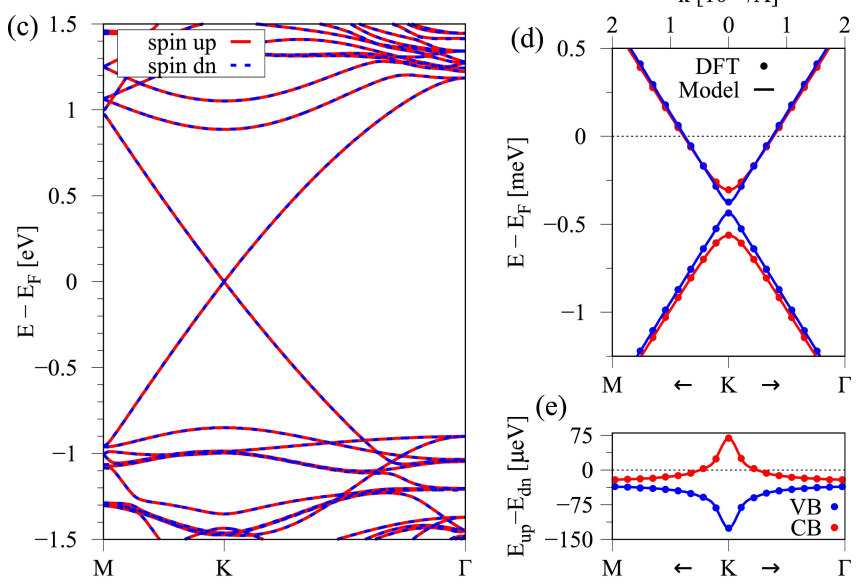

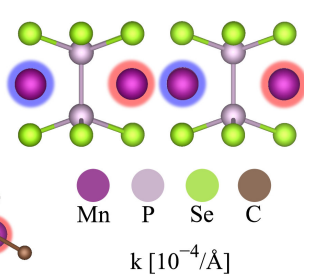

FIG. 3. (a,b) Top and side view of graphene/MnPSe 3 heterostructure. The colored spheres around $\mathrm{Mn}$ atoms indicate the magnetization direction (red spin up, blue spin down) with Ising-type magnetic ordering. (c) Calculated electronic band structure of graphene/ $\mathrm{MnPSe}_{3}$ heterostructure without SOC (to demonstrate bare staggered exchange) for an interlayer distance of $3.36 \AA$. Bands in red (blue) correspond to spin up (down). (d) Enlargement of the calculated low energy bands (symbols) at $\mathrm{K}$ with a fit to model Hamiltonian $\mathcal{H}_{\mathrm{GR}}$ 52] (solid line). (e) The splitting between spin up and spin down bands $\mathrm{E}_{\mathrm{up}}-\mathrm{E}_{\mathrm{dn}}$ for the $\mathrm{VB}$ and $\mathrm{CB}$. 

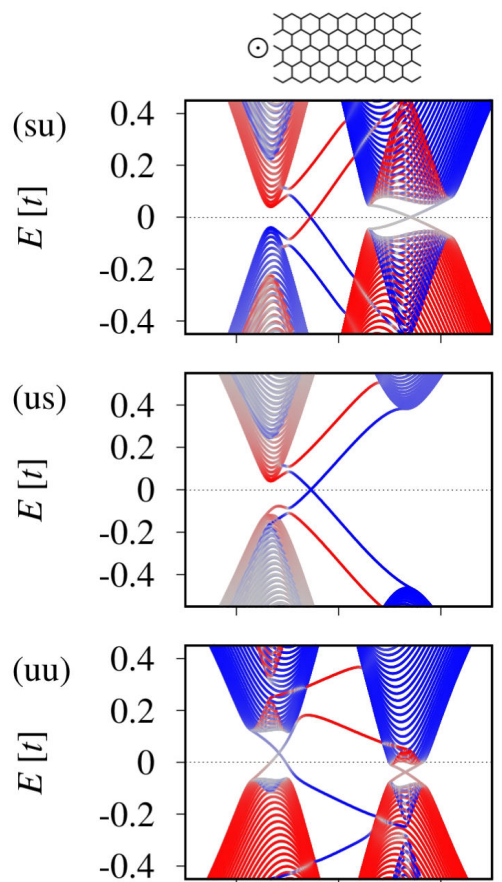

(ss)

$\pm$
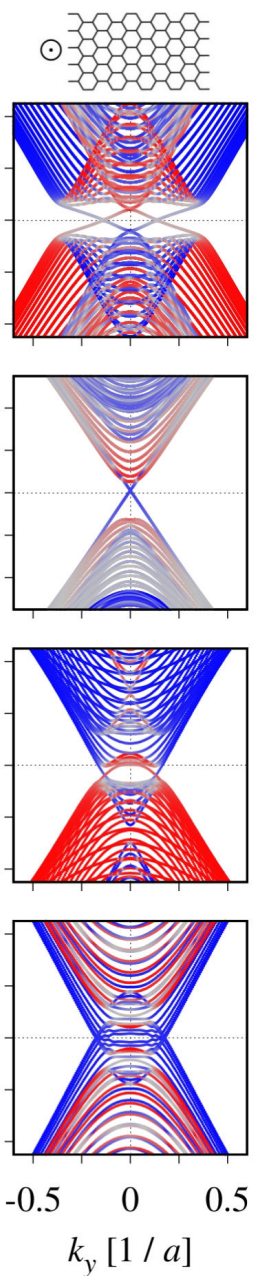
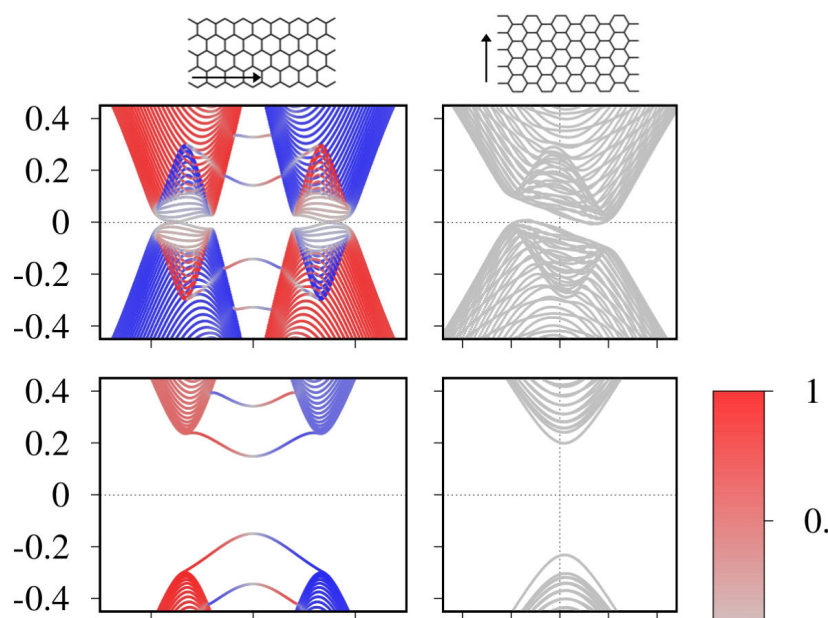

0.5
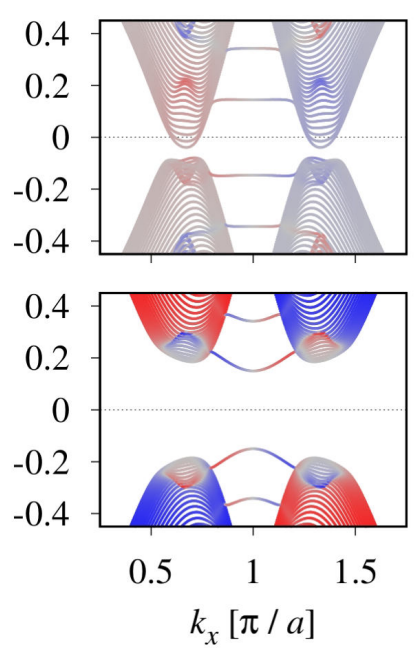

0
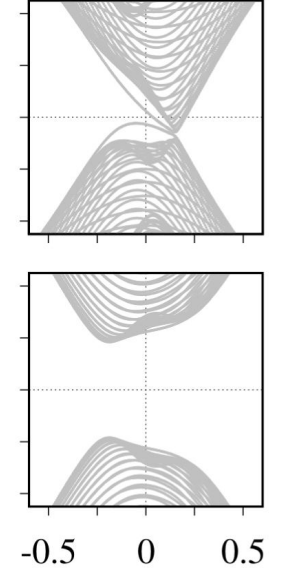

$k_{y}[1 / a]$

FIG. 4. Calculated electronic structure of zigzag and armchair 100 unit cells wide nanoribbons. Arrows specify direction of magnetization, parallel to the $z$-axis $(\theta=0, \phi=0)$ in the left two columns and parallel to the $x$-axis $(\theta=\pi / 2, \phi=0)$ in the right two columns. Color indicates $\hat{s}_{z}$ spin expectation value. We use $\Delta=0.1 t, \lambda_{R}=(3 / 2) \times 0.05 t, \lambda_{E}^{A}=\left|\lambda_{E}^{B}\right|=0.25 t$ and $\lambda_{I}^{A}=\left|\lambda_{I}^{B}\right|=3 \sqrt{3} \times 0.06 t$ in (su), (us), (ss), and $\lambda_{I}^{A}=\left|\lambda_{I}^{B}\right|=0.1 t$ in (uu).

of the low energy model Hamiltonian [52, for several interlayer distances, show that the sublattice resolved proximity exchange parameters have opposite signs.

Nanoribbons. We now go back to the model investigations. Due to bulk-edge correspondence the Chern number gives the number of topological states that appear per edge in a nanoribbon. For zigzag and armchair termination this is displayed in the first and second column of Fig. 4, respectively. The nanoribbon spectra confirm the presence of chiral edge states in the QAHE phases (su), (us), and (uu) [52. In a finite flake the states of both termination types connect and travel along the edges as schematically depicted for cases (su) and (us) in Figs. 1(b) and 1(c). The (su) case is curious. Without exchange, this case is topologically trivial, but protected pseudohelical states emerge in a finite ribbon [8] along one zigzag edge the state has spin up, along the opposite one it has spin down. Spin flip occurs in tunneling along an armchair edge. The degenerate, time-reversed partners show analog behavior. The breaking of the timereversal symmetry makes the system topological and the pseudohelical states, depicted in Fig. 1.(b), become propagating also along the armchair direction. Thus, in a QAHE regime, we predict protected edge states whose spin polarization is opposite in opposite zigzag edges.

Despite their chiral nature the QAHE states differ in their localization behavior and spin. The states' position in $\mathbf{k}$-space is crucial for both. Inside a cone the edge states are much closer to bulk states and so have a significantly larger decay length [e. g., case (uu) zigzag ribbon] compared to the ones that reside between two cones [e.g., case (us) zigzag ribbon] (see Supplemental Material [52]). In addition, their spin polarization gets blurred inside the valleys.

The zigzag ribbon in case (su) combines both types of states and allows to tune the spin-polarized interval- 
ley modes to unpolarized intravalley states by decreasing intrinsic SOC to zero. Note that for, $\lambda_{I}=0$, cases $(\mathrm{su})$ and (uu) coincide and reproduce an earlier model for QAHE states in graphene based on Rashba SOC and exchange, which has two intravalley states in each valley [7, 9]. Remarkably, the intervalley modes, which are stabilized by intrinsic SOC, are much more localized than the intravalley states. Therefore, the intervalley states are more robust against weak disorder. But the long localization length of the intravalley states offers the possibility to tune the case (su) by finite size effects. While the probability amplitude of intervalley states falls off to zero within a few lattice sites, the wave function of intravalley states extends over more than 30 sites (see Supplemental Material [52]). In a small ribbon the latter will hybridize and be gapped out so that we are left with only one state per edge. Similar scenario occurs in the pseudohelical regime at quantum spin Hall effect [8].

In the armchair ribbon $\mathrm{K}$ and $\mathrm{K}^{\prime}$ points are folded back to $\Gamma$ point, thus all edge states are within the valleys and are less localized compared to zigzag edges due to the vicinity to the bulk spectrum [52].

Magnetic anisotropy of QAHE. Finally, we demonstrate that the topological order and edge states can be controlled by magnetization orientation. We direct the exchange to point in plane along $x$; for uniform exchange this geometry was considered in Ref. 58. It is clear from the presented results in Fig. 4 (third and fourth columns), that in all four cases the system is in a trivial phase after the rotation. This result is elaborated on in the Supplemental Material [52, where we identify trivial insulator or metallic regions in topological diagrams, and also show the evolution of bulk and ribbon bands under rotation of magnetization. When magnetization is induced to graphene via a small external magnetic field, as is possible in cases (su) and (uu), rotating the magnetic field can be used to perform a topological phase transition where all other system parameters remain the same.

In summary, a comprehensive parameter space analysis of a realistic effective model with combinations of uniform and staggered spin-orbit and exchange proximitized graphene is presented, predicting magnetization orientation dependent QAHE topological phases and edge states. A specific material platform for staggered exchange in graphene is proposed, based on DFT studies of graphene on Ising antiferromagnetic $\mathrm{MnPSe}_{3}$.

This work has been funded by the Deutsche Forschungsgemeinschaft (DFG, German Research Foundation) Project-No. 314695032 SFB 1277, DFG SPP 1666, EU Seventh Framework Programme under Grant Agreements No. 604391 Graphene Flagship, No. VVGS2018-1227, and No. VEGA 1/0105/20.
* Emails to: petra.hoegl@physik.uni-regensburg.de

[1] Y. Ren, Z. Qiao, and Q. Niu, Rep. Prog. Phys. 79, 066501 (2016)

[2] I. Zutić, J. Fabian, and S. Das Sarma, Rev. Mod. Phys. 76, 323 (2004).

[3] W. Han, R. K. Kawakami, M. Gmitra, and J. Fabian, Nat. Nano 9, 794 (2014).

[4] F. D. M. Haldane, Phys. Rev. Lett. 61, 2015 (1988)

[5] C. L. Kane and E. J. Mele, Phys. Rev. Lett. 95, 226801 (2005).

[6] C. L. Kane and E. J. Mele, Phys. Rev. Lett. 95, 146802 (2005).

[7] Z. Qiao, S. A. Yang, W. Feng, W.-K. Tse, J. Ding, Y. Yao, J. Wang, and Q. Niu, Phys. Rev. B 82, 161414(R) (2010)

[8] T. Frank, P. Högl, M. Gmitra, D. Kochan, and J. Fabian, Phys. Rev. Lett. 120, 156402 (2018)

[9] Z. Qiao, H. Jiang, X. Li, Y. Yao, and Q. Niu, Phys. Rev. B 85, 115439 (2012)

[10] J. Zhang, B. Zhao, Y. Yao, and Z. Yang, Phys. Rev. B 92, 165418 (2015).

[11] J. Zhang, B. Zhao, Y. Yao, and Z. Yang, Scientific Reports 5, 10629 (2015).

[12] Z. Zanolli, C. Niu, G. Bihlmayer, Y. Mokrousov, P. Mavropoulos, M. J. Verstraete, and S. Blügel, Phys. Rev. B 98, 155404 (2018).

[13] J. Zhang, B. Zhao, T. Zhou, Y. Xue, C. Ma, and Z. Yang, Phys. Rev. B 97, 085401 (2018).

[14] S. Su, Y. Barlas, J. Li, J. Shi, and R. K. Lake, Phys. Rev. B 95, 075418 (2017).

[15] A. K. Geim and I. V. Grigorieva, Nature 499, 419 (2013),

[16] M. Gmitra, S. Konschuh, C. Ertler, C. Ambrosch-Draxl, and J. Fabian, Phys. Rev. B 80, 235431 (2009).

[17] J. Sichau, M. Prada, T. Anlauf, T. J. Lyon, B. Bosnjak, L. Tiemann, and R. H. Blick, Phys. Rev. Lett. 122, 046403 (2019).

[18] M. Gmitra and J. Fabian, Phys. Rev. B 92, 155403 (2015).

[19] M. Gmitra, D. Kochan, P. Högl, and J. Fabian, Phys. Rev. B 93, 155104 (2016).

[20] Z. Wang, D.-K. Ki, H. Chen, H. Berger, A. H. MacDonald, and A. F. Morpurgo, Nat. Commun. 6, 8339 (2015).

[21] B. Yang, M.-F. Tu, J. Kim, Y. Wu, H. Wang, J. Alicea, R. Wu, M. Bockrath, and J. Shi, 2D Mater. 3, 031012 (2016)

[22] Z. Wang, D.-K. Ki, J. Y. Khoo, D. Mauro, H. Berger, L. S. Levitov, and A. F. Morpurgo, Phys. Rev. X 6, $041020(2016)$

[23] T. Völkl, T. Rockinger, M. Drienovsky, K. Watanabe, T. Taniguchi, D. Weiss, and J. Eroms, Phys. Rev. B 96, 125405 (2017)

[24] A. Avsar, J. Y. Tan, T. Taychatanapat, J. Balakrishnan, G. K. W. Koon, Y. Yeo, J. Lahiri, A. Carvalho, A. S. Rodin, E. C. T. O'Farrell, G. Eda, A. H. Castro Neto, and B. Özyilmaz, Nat. Commun. 5, 4875 (2014).

[25] S. Omar and B. J. van Wees, Phys. Rev. B 95, 081404(R) (2017).

[26] A. Dankert and S. P. Dash, Nat. Commun. 8, 16093 (2017)

[27] M. Offidani, M. Milletarì, R. Raimondi, and A. Ferreira, Phys. Rev. Lett. 119, 196801 (2017) 
[28] T. P. Kaloni, L. Kou, T. Frauenheim, and U. Schwingenschlögl, Appl. Phys. Lett. 105, 233112 (2014).

[29] S. Zihlmann, A. W. Cummings, J. H. Garcia, M. Kedves, K. Watanabe, T. Taniguchi, C. Schönenberger, and P. Makk, Phys. Rev. B 97, 075434 (2018).

[30] T. S. Ghiasi, J. Ingla-Aynés, A. A. Kaverzin, and B. J. van Wees, Nano Letters 17, 7528 (2017).

[31] A. W. Cummings, J. H. Garcia, J. Fabian, and S. Roche, Phys. Rev. Lett. 119, 206601 (2017).

[32] L. A. Benítez, J. F. Sierra, W. S. Torres, A. Arrighi, F. Bonell, M. V. Costache, and S. O. Valenzuela, Nature Physics 14, 303 (2018).

[33] K. Zollner, M. Gmitra, T. Frank, and J. Fabian, Phys. Rev. B 94, 155441 (2016)

[34] P. Lazić, K. D. Belashchenko, and I. Žutić, Phys. Rev. B 93, 241401(R) (2016)

[35] Z. Wang, C. Tang, R. Sachs, Y. Barlas, and J. Shi, Phys. Rev. Lett. 114, 016603 (2015)

[36] J. C. Leutenantsmeyer, A. A. Kaverzin, M. Wojtaszek, and B. J. van Wees, 2D Mater. 4, 014001 (2017)

[37] J. B. S. Mendes, O. Alves Santos, L. M. Meireles, R. G. Lacerda, L. H. Vilela-Leão, F. L. A. Machado, R. L. Rodríguez-Suárez, A. Azevedo, and S. M. Rezende, Phys. Rev. Lett. 115, 226601 (2015).

[38] A. G. Swartz, P. M. Odenthal, Y. Hao, R. S. Ruoff, and R. K. Kawakami, ACS Nano 6, 10063 (2012)

[39] P. Wei, S. Lee, F. Lemaitre, L. Pinel, D. Cutaia, W. Cha, F. Katmis, Y. Zhu, D. Heiman, J. Hone, J. S. Moodera, and C.-T. Chen, Nature Materials 15, 711 (2016).

[40] H. Haugen, D. Huertas-Hernando, and A. Brataas, Phys. Rev. B 77, 115406 (2008)

[41] H. X. Yang, A. Hallal, D. Terrade, X. Waintal, S. Roche, and M. Chshiev, Phys. Rev. Lett. 110, 046603 (2013).

[42] A. Hallal, F. Ibrahim, H. Yang, S. Roche, and M. Chshiev, 2D Mater. 4, 025074 (2017)

[43] A. Dyrda and J. Barna, 2D Mater. 4, 034003 (2017)

[44] K. Zollner, M. Gmitra, and J. Fabian, New Journal of Physics 20, 073007 (2018)

[45] D. Kochan, S. Irmer, and J. Fabian, Phys. Rev. B 95, 165415 (2017)

[46] M. Gmitra, D. Kochan, and J. Fabian, Phys. Rev. Lett. 110, 246602 (2013)

[47] J. W. McClure and Y. Yafet, Proceedings of the Fifth Conference on Carbon 1, 22 (1962)

[48] D. J. Thouless, M. Kohmoto, M. P. Nightingale, and
M. den Nijs, Phys. Rev. Lett. 49, 405 (1982).

[49] S. Ryu, A. P. Schnyder, A. Furusaki, and A. W. W. Ludwig, New J. Phys. 12, 065010 (2010).

[50] A. Marrazzo, M. Gibertini, D. Campi, N. Mounet, and N. Marzari, Phys. Rev. Lett. 120, 117701 (2018).

[51] K. Song, D. Soriano, A. W. Cummings, R. Robles, P. Ordejn, and S. Roche, Nano Letters 18, 2033 (2018).

[52] See Supplemental Material at [URL will be inserted by publisher] for more details, which includes Refs. [59 68].

[53] M. Luo, Phys. Rev. B 99, 165407 (2019).

[54] Q. Pei, X. Wang, J. Zou, and W. Mi, Front. Phys. 13, 137105 (2018).

[55] A. Wiedenmann, J. Rossat-Mignod, A. Louisy, R. Brec, and J. Rouxel, Solid State Commun. 40, 1067 (1981).

[56] V. Grasso and L. Silipigni, J. Opt. Soc. Am. B 16, 132 (1999)

[57] Z. Qiao, W. Ren, H. Chen, L. Bellaiche, Z. Zhang, A. H. MacDonald, and Q. Niu, Phys. Rev. Lett. 112, 116404 (2014)

[58] Y. Ren, J. Zeng, X. Deng, F. Yang, H. Pan, and Z. Qiao, Phys. Rev. B 94, 085411 (2016)

[59] P. Hohenberg and W. Kohn, Phys. Rev. 136, B864 (1964)

[60] P. Giannozzi, S. Baroni, N. Bonini, M. Calandra, R. Car, C. Cavazzoni, D. Ceresoli, G. L. Chiarotti, M. Cococcioni, I. Dabo, A. D. Corso, S. Fabris, G. Fratesi, S. de Gironcoli, R. Gebauer, U. Gerstmann, C. Gougoussis, A. Kokalj, M. Lazzeri, L. Martin-Samos, N. Marzari, F. Mauri, R. Mazzarello, S. Paolini, A. Pasquarello, L. Paulatto, C. Sbraccia, S. Scandolo, G. Sclauzero, A. P. Seitsonen, A. Smogunov, P. Umari, and R. M. Wentzcovitch, J. Phys.: Condens. Mat. 21, 395502 (2009)

[61] Q. Pei, Y. Song, X. Wang, J. Zou, and W. Mi, Sci. Rep. 7, 9504 (2017)

[62] Q. Pei, X. Wang, J. Zou, and W. Mi, Nanotechnology 29, 214001 (2018).

[63] G. Kresse and D. Joubert, Phys. Rev. B 59, 1758 (1999).

[64] J. P. Perdew, K. Burke, and M. Ernzerhof, Phys. Rev. Lett. 77, 3865 (1996).

[65] S. Grimme, J. Comput. Chem. 27, 1787 (2006).

[66] V. Barone, M. Casarin, D. Forrer, M. Pavone, M. Sambi, and A. Vittadini, J. Comput. Chem. 30, 934 (2009)

[67] Y. Baskin and L. Meyer, Phys. Rev. 100, 544 (1955)

[68] V. T. Phong, N. R. Walet, and F. Guinea, 2D Mater. 5, $014004(2017)$ 


\title{
SUPPLEMENTAL MATERIAL Quantum Anomalous Hall Effects in Graphene from Proximity-Induced Uniform and Staggered Spin-Orbit and Exchange Coupling
}

\author{
Petra Högl, ${ }^{1, *}$ Tobias Frank, ${ }^{1}$ Klaus Zollner,${ }^{1}$ Denis Kochan,,${ }^{1}$ Martin Gmitra, ${ }^{2}$ and Jaroslav Fabian ${ }^{1}$ \\ ${ }^{1}$ Institute for Theoretical Physics, University of Regensburg, 93040 Regensburg, Germany \\ ${ }^{2}$ Department of Theoretical Physics and Astrophysics, \\ Pavol Jozef Šafárik University, 04001 Košice, Slovakia
}

Throughout the paper we consider the following four possible combinations of intrinsic spin-orbit and exchange coupling: uniform-uniform (uu) $\lambda_{I}^{A}=\lambda_{I}^{B}$ and $\lambda_{E}^{A}=\lambda_{E}^{B}$, uniform-staggered (us) $\lambda_{I}^{A}=\lambda_{I}^{B}$ and $\lambda_{E}^{A}=$ $-\lambda_{E}^{B}$, staggered-uniform (su) $\lambda_{I}^{A}=-\lambda_{I}^{B}$ and $\lambda_{E}^{A}=\lambda_{E}^{B}$, and staggered-staggered (ss) $\lambda_{I}^{A}=-\lambda_{I}^{B}$ and $\lambda_{E}^{A}=-\lambda_{E}^{B}$. We fix the model parameters to generic values $\Delta=0.1 t$ and $\lambda_{R}=(3 / 2) \times 0.05 t$. In the Supplemental Material, we discuss additional information to these four combinations of staggered and uniform parameters, provide details to the first-principles calculations, and investigate intermediate cases for which we relax the staggered or uniform condition for the intrinsic spin-orbit coupling or exchange coupling parameters.

\section{Chern number}

The Chern number is defined as $[1,2]$

$$
C=\frac{1}{2 \pi} \sum_{m}^{o c c} \int_{B Z} d^{2} \mathbf{k} \Omega_{z, \mathbf{k}}^{m}
$$

where $\Omega_{z, \mathbf{k}}^{m}$ is the $z$-component of the Berry curvature for the $m$-th band in momentum space

$\Omega_{z, \mathbf{k}}^{m}=\sum_{n \neq m} \frac{-2 \operatorname{Im}\left\langle u_{\mathbf{k}}^{m}\left|\partial_{k_{x}} H_{\mathbf{k}}\right| u_{\mathbf{k}}^{n}\right\rangle\left\langle u_{\mathbf{k}}^{n}\left|\partial_{k_{y}} H_{\mathbf{k}}\right| u_{\mathbf{k}}^{m}\right\rangle}{\left(E_{\mathbf{k}}^{m}-E_{\mathbf{k}}^{n}\right)^{2}}$

The Chern number is calculated from the Fourier transformed Hamiltonian $H_{\mathbf{k}}$ with the periodic part of Bloch wavefunctions $u_{\mathbf{k}}^{m}$ and eigenenergies $E_{\mathbf{k}}^{m}$. Summation is over all occupied bands $(m=1,2)$ and integration over the Brillouin zone.

\section{Analytical conditions for gap closings}

In the main paper we numerically compute the bulk band gap and Chern number to characterize the topological phase space. Additionally, we provide analytical conditions to be in a nontrivial phase, determined from direct gap closing at $\mathrm{K}$ and $\mathrm{K}^{\prime}$ or indirect gap closing between bands at $\mathrm{K}$ and $\mathrm{K}^{\prime}$,

$$
\begin{aligned}
& \text { (su) }\left|\lambda_{E}\right|>\Delta-\lambda_{I} \wedge\left|\lambda_{E}\right|>\lambda_{I}-\Delta, \\
& \text { (us) }\left|\lambda_{E}\right|>\left|\lambda_{I}\right|-\frac{1}{2} \sqrt{\Delta^{2}+4 \lambda_{R}^{2}}-\frac{\Delta}{2} \\
& \wedge\left|\lambda_{E}\right|>-\left|\lambda_{I}\right|+\frac{1}{2} \sqrt{\Delta^{2}+4 \lambda_{R}^{2}}+\frac{\Delta}{2} \\
& \wedge \text { (S4) } \\
& \wedge<\left|\lambda_{E}\right| \\
& \wedge\left|\lambda_{I}\right|+\frac{1}{2} \sqrt{\Delta^{2}+4 \lambda_{R}^{2}}+\frac{\Delta}{2} \sqrt{\Delta^{2}+4 \lambda_{R}^{2}}-\frac{\Delta}{2}, \\
& \text { (uu) }\left|\lambda_{I}\right|<\frac{\left|\lambda_{E}\right|}{2}+\frac{1}{2} \sqrt{\left(\Delta-\left|\lambda_{E}\right|\right)^{2}+4 \lambda_{R}^{2}}-\frac{\Delta}{2} \text { (S5) } \\
& \wedge\left|\lambda_{E}\right|>\Delta,
\end{aligned}
$$

with $\left|\lambda_{I}\right|=\left|\lambda_{I}^{A}\right|=\left|\lambda_{I}^{B}\right|$ and $\left|\lambda_{E}\right|=\left|\lambda_{E}^{A}\right|=\left|\lambda_{E}^{B}\right|$. For (su) and (us) they agree exactly with the numerically found global gap closings in Fig. 2 in the main text. Case (uu) is an extension of Ref. [3] including both staggered potential and intrinsic spin-orbit coupling (SOC), and agrees with their results for $\Delta=0$ and $\lambda_{I}=0$. In this case quantum anomalous Hall effect (QAHE) originates from the interplay of exchange splitting and Rashba SOC. Increasing intrinsic SOC from zero to a finite value along the horizontal axis in Fig. 2(uu) in the main paper shows the stability of the QAHE state against weak intrinsic SOC at fixed exchange coupling in the presence of staggered potential $\Delta$. The first expression in Eq. (S5) gives an approximate limit for $\lambda_{I}$. For small intrinsic SOC it is an exact condition and corresponds to an indirect gap closing between $\mathrm{K}$ and $\mathrm{K}^{\prime}$. At $\lambda_{I} \gtrsim \Delta$ the bulk gap closes in the vicinity of K-points and narrows the topological region compared to the one specified by the first term in Eq. (S5). Cases (su) and (uu) additionally require finite Rashba SOC to have a gapped system in the vicinity of the Dirac points.

\section{Pseudospin and spin texture in k-space}

We use the Berry curvature to compute the Chern numbers and determine QAHE phases in the main paper. Here we want to connect the Berry curvature and resulting Chern numbers to the pseudospin and real spin textures in $\mathbf{k}$-space. 
TABLE S1. Chern numbers $C_{\mathrm{K} / \mathrm{K}^{\prime}}^{m}$ per valley and band $m$ and total Chern number $C$ for parameters $\lambda_{E}^{A}=\left|\lambda_{E}^{B}\right|=0.25 t$ and $\lambda_{I}^{A}=\left|\lambda_{I}^{B}\right|=3 \sqrt{3} \times 0.06 t$ in (su), (us), and $\lambda_{I}^{A}=\left|\lambda_{I}^{B}\right|=0.1 t$ in (uu).

\begin{tabular}{c|cccc|c}
\hline \hline & $C_{\mathrm{K}}^{2}$ & $C_{\mathrm{K}^{\prime}}^{2}$ & $C_{\mathrm{K}}^{1}$ & $C_{\mathrm{K}^{\prime}}^{1}$ & $C$ \\
\hline (su) & 1.54 & 1.46 & -0.46 & -0.54 & 2 \\
\hline (us) & 0.149 & -0.144 & -0.0746 & 1.07 & 1 \\
\hline (uu) & 1.54 & 0.46 & -0.46 & 0.46 & 2 \\
\hline \hline
\end{tabular}

Berry curvature can be considered as analog of magnetic field in $\mathbf{k}$-space [4]. Thus, a finite Berry curvature at momentum $\mathbf{k}$ means that the spin degree of freedom starts to wind at this point in momentum space. The winding can either form a skyrmion or a meron. A skyrmion can be built from the combination of a meron and an anti-meron. Skyrmions and merons are topologically protected and carry a topological charge of \pm 1 and $\pm 1 / 2$, respectively, which is also called their winding number. The sum of the topological charges from the valence bands (VBs) is equal to the Chern number.

In graphene we have two spin-like degrees of freedom: pseudospin $\hat{\boldsymbol{\sigma}}$ and real spin $\hat{\mathbf{s}}$. In our effective 4 -band model with the basis $\left(u_{\mathbf{k}, A \uparrow}^{m}, u_{\mathbf{k}, A \downarrow}^{m}, u_{\mathbf{k}, B \uparrow}^{m}, u_{\mathbf{k}, B \downarrow}^{m}\right)$ they are coupled. To map their texture in momentum space we compute the expectation value of pseudospin and spin component $j=x, y, z$ projected on the respective subspace for the $m$-th band

$$
\begin{aligned}
\langle\hat{\boldsymbol{\sigma}}\rangle_{\mathbf{k}}^{m} & =\sum_{j}\left\langle u_{\mathbf{k}}^{m}\left|\hat{\sigma}_{j} \otimes \mathbb{1}_{s}\right| u_{\mathbf{k}}^{m}\right\rangle \hat{\mathbf{e}}_{j}, \\
\langle\hat{\mathbf{s}}\rangle_{\mathbf{k}}^{m} & =\sum_{j}\left\langle u_{\mathbf{k}}^{m}\left|\mathbb{1}_{\sigma} \otimes \hat{s}_{j}\right| u_{\mathbf{k}}^{m}\right\rangle \hat{\mathbf{e}}_{j},
\end{aligned}
$$

with unit vector $\hat{\mathbf{e}}_{j}$. In Figs. S1-S3, we show vector plots of $\langle\hat{\boldsymbol{\sigma}}\rangle_{\mathbf{k}}^{m}$ and $\langle\hat{\boldsymbol{s}}\rangle_{\mathbf{k}}^{m}$ for the two VBs $(m=1$ low energy VB, $m=2$ high energy VB) for cases (su), (us), and (uu) from which we want to identify skyrmion and meron structures. Further, we compute the Chern numbers $C_{\mathrm{K} / \mathrm{K}^{\prime}}^{m}$ per valley and band $m=1,2$ where

$$
C=\sum_{m}^{o c c} C_{\mathrm{K}}^{m}+C_{\mathrm{K}^{\prime}}^{m} .
$$

The Chern numbers are summarized in Table S1. The Berry curvature is discussed in the next section.

The Chern numbers per valley and band contain contributions from both pseudospin and spin and are not quantized. We now want to analyze how they are combined. In case (su), shown in Fig. S1, we see that both VBs contribute. The pseudospin texture has a meron in each valley in both VBs. The pseudospin textures in $\mathrm{K}$ correspond to anti-merons, the ones in $\mathrm{K}^{\prime}$ to merons. The spin texture in the second VB provides one skyrmion in

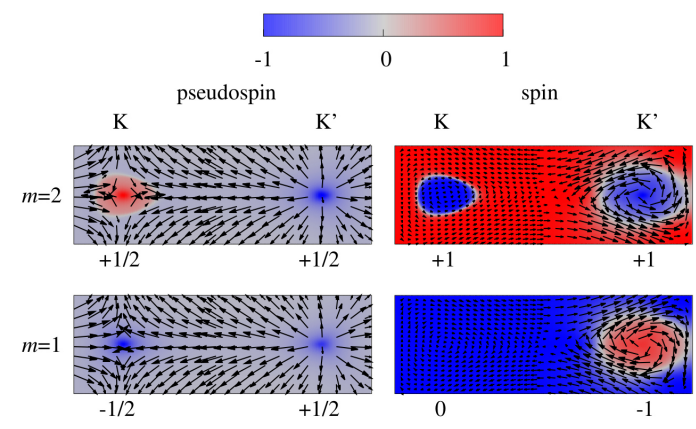

FIG. S1. Case (su). Pseudospin texture $\langle\hat{\boldsymbol{\sigma}}\rangle_{\mathbf{k}}^{m}$ (left column) and spin texture $\langle\hat{\mathbf{s}}\rangle_{\mathbf{k}}^{m}$ (right column) in $\mathbf{k}$-space around $\mathrm{K}$ and $\mathrm{K}^{\prime}$. Color indicates $z$-component, arrows are a $3 \mathrm{~d}$ vector plot. Top panels show top VB $(m=2)$, bottom panels present bottom VB $(m=1)$. The numbers indicate topological charges. Parameters are: $\lambda_{I}^{A}=\left|\lambda_{I}^{B}\right|=3 \sqrt{3} \times 0.06 t$ and $\lambda_{E}^{A}=\left|\lambda_{E}^{B}\right|=0.25 t$ with $\lambda_{I}^{A}=-\lambda_{I}^{B}$ and $\lambda_{E}^{A}=\lambda_{E}^{B}$.

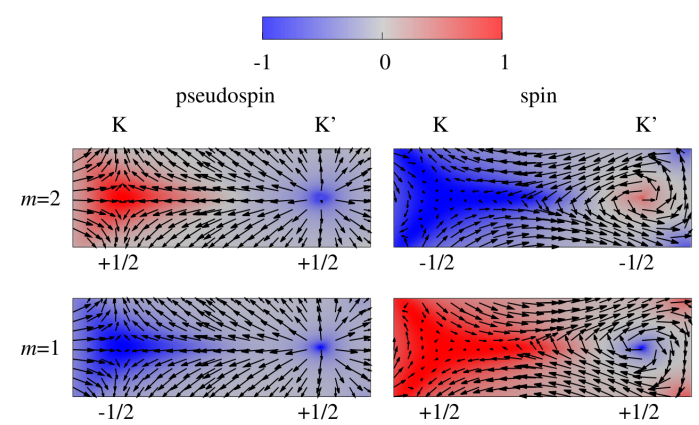

FIG. S2. Case (us). Pseudospin texture $\langle\hat{\boldsymbol{\sigma}}\rangle_{\mathbf{k}}^{m}$ (left column) and spin texture $\langle\hat{\mathbf{s}}\rangle_{\mathbf{k}}^{m}$ (right column) in $\mathbf{k}$-space around $\mathrm{K}$ and $\mathrm{K}^{\prime}$. Color indicates $z$-component, arrows are a $3 \mathrm{~d}$ vector plot. Top panels show top VB $(m=2)$, bottom panels present bottom VB $(m=1)$. The numbers indicate topological charges. Parameters are: $\lambda_{I}^{A}=\left|\lambda_{I}^{B}\right|=3 \sqrt{3} \times 0.06 t$ and $\lambda_{E}^{A}=\left|\lambda_{E}^{B}\right|=0.25 t$ with $\lambda_{I}^{A}=\lambda_{I}^{B}$ and $\lambda_{E}^{A}=-\lambda_{E}^{B}$.

$\mathrm{K}$ and one in $\mathrm{K}^{\prime}$, contrary for the first VB we have only one skyrmion in $\mathrm{K}^{\prime}$. If we sum the topological charges over the VBs we find that the contributions from the two pseudospin merons in $\mathrm{K}$ as well as the two spin skyrmions in $\mathrm{K}^{\prime}$ cancel each other, respectively. Whereas the two merons in $\mathrm{K}^{\prime}$ and the skyrmion in $\mathrm{K}$ remain and form the QAHE state. From their topological charge follows Chern number $C_{\mathrm{K}}=1$ and $C_{\mathrm{K}^{\prime}}=1$ in the two valleys resulting in the total Chern number $C=2$. From this consideration we can understand the numerically calculated Chern numbers per band and valley in Table S1 which are the combinations of the contributions from pseudospin and spin. They are not exactly (half-)quantized because we have an allowed asymmetry in $\mathrm{K}$ and $\mathrm{K}^{\prime}$ due to the broken time-reversal symmetry. The total Chern num- 


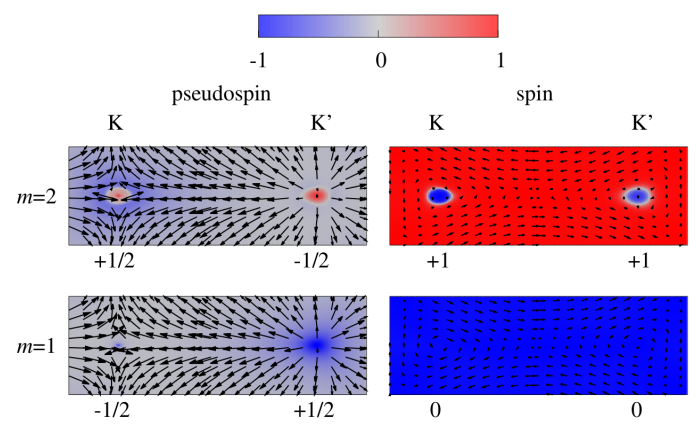

FIG. S3. Case (uu). Pseudospin texture $\langle\hat{\boldsymbol{\sigma}}\rangle_{\mathbf{k}}^{m}$ (left column) and spin texture $\langle\hat{\mathbf{s}}\rangle_{\mathbf{k}}^{m}$ (right column) in $\mathbf{k}$-space around $\mathrm{K}$ and $\mathrm{K}^{\prime}$. Color indicates $z$-component, arrows are a $3 \mathrm{~d}$ vector plot. Top panels show top VB $(m=2)$, bottom panels present bottom VB $(m=1)$. The numbers indicate topological charges. Parameters are: $\lambda_{I}^{A}=\left|\lambda_{I}^{B}\right|=0.1 t$ and $\lambda_{E}^{A}=\left|\lambda_{E}^{B}\right|=0.25 t$ with $\lambda_{I}^{A}=\lambda_{I}^{B}$ and $\lambda_{E}^{A}=\lambda_{E}^{B}$.

ber is quantized and we get $C=2$ (as given in the main paper).

In case (us) we find two pseudospin anti-merons in $\mathrm{K}$ and two merons in $\mathrm{K}^{\prime}$. Spin also forms two anti-merons in $\mathrm{K}$ and two merons in $\mathrm{K}^{\prime}$ (Fig. S2). All contributions except the two merons in the first VB in $\mathrm{K}^{\prime}$ cancel each other and we find them to cause the QAHE state with Chern number $C=1$.

The last case for which we found QAHE states is (uu) which is shown in Fig. S3. This is an extension of Ref. [3], where we consider both uniform intrinsic SOC and staggered potential. Similar to their results, we get two pseudospin anti-merons in $\mathrm{K}$ and two merons in $\mathrm{K}^{\prime}$ (one in each band), whose topological charges cancel each other when summing over the VBs. From spin in the second $\mathrm{VB}$ at $\mathrm{K}$ and $\mathrm{K}^{\prime}$ we find two skyrmions. This leads to $C_{\mathrm{K}}=1$ and $C_{\mathrm{K}^{\prime}}=1$ resulting in the total Chern number $C=2$. This phenomenological examination is in agreement with the computed Chern numbers per band and valley in Table S1.

\section{Bulk band structure}

We now investigate the Berry curvature defined in Eq. (S2) and the bulk band structures in Fig. S4 at representative points in the phase diagrams. In Fig. S4, skyrmions can be identified from spin resolved band structure plots by the evolution of spin expectation value red-gray-blue-gray-red or blue-gray-red-gray-blue in the VBs; (anti-)merons from changing pseudospin or spin expectation value as gray-red-gray or gray-blue-gray in the Berry curvature or band structure plot, respectively. Skyrmions and merons are accompanied by a finite Berry curvature. Its sign allows to read off the sign of the

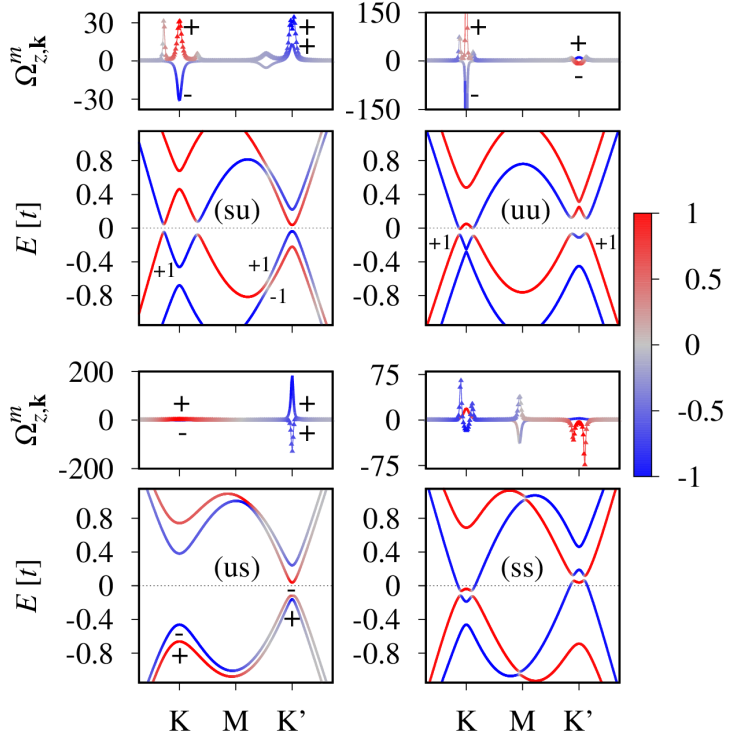

FIG. S4. Calculated Berry curvature of first (solid line) and second (line with triangles) VB and bulk band structure along $k_{y}=0$. Color indicates $\hat{\sigma}_{z}$ pseudospin expectation value in Berry curvature plots and $\hat{s}_{z}$ spin expectation value in band structure plots. Merons are denoted by $+(-)$ with topological charge $+1 / 2(-1 / 2)$ stemming from pseudospin (Berry curvature plot) or spin (band structure plot) texture. The skyrmions from spin texture are specified by \pm 1 with respective topological charge \pm 1 . We use $\lambda_{E}^{A}=\left|\lambda_{E}^{B}\right|=0.25 t$ and $\lambda_{I}^{A}=\left|\lambda_{I}^{B}\right|=3 \sqrt{3} \times 0.06 t$ in (su), (us), (ss), and $\lambda_{I}^{A}=\left|\lambda_{I}^{B}\right|=0.1 t$ in (uu)

topological charge that we can assign to the nontrivial (pseudo)spin textures, namely \pm 1 for skyrmions and $\pm 1 / 2$ for merons. The sum of all topological charges in the VBs equals the Chern number. Table S1 summarizes the numerical Chern numbers per band and valley.

\section{Localization of edge states}

Bulk-boundary correspondence states the appearance of chiral edge states in a nanoribbon if the bulk has a finite Chern number. The number of states per edge is given by that Chern number. We show the presence of states within the bulk band gap for zigzag and armchair edges for cases (su), (us), and (uu) in the main paper. Here, we explicitly check their chiral nature, i.e., a fixed relation between direction of propagation and side of the ribbon on which they propagate. For this we sum the probability amplitude in the $i$-th unit cell of a state $\psi_{i}^{m}(k)$ weighted by the position of the unit cell in the 
ribbon

$$
\sum_{i=0}^{N}\left(i-\frac{N}{2}\right)\left|\psi_{i}^{m}(k)\right|^{2} / \frac{N}{2},
$$

where $N$ is the number of unit cells and $k$ is the momentum ( $k=k_{x}$ for zigzag, $k=k_{y}$ for armchair ribbon). From the resulting number that varies between -1 and 1 we learn on which side of the ribbon a state propagates and how strong it is confined to the edge. In Fig. S5 we plot the bands for zigzag and armchair ribbons with their localization behavior. Inside the bulk band gap, the states are in all three cases localized for both types of edge termination. On which side of the ribbon they propagate can be read off the scheme in Fig. S5. In the zigzag (armchair) ribbon, the modes with negative velocity $v_{k}^{m}=\partial_{k} E_{k}^{m} / \hbar$ are localized at $y=0(x=W)$ while the ones with positive velocity are at $y=L(x=0)$. So they form chiral states along the two zigzag edges as well as the two armchair edges of the respective infinite nanoribbons. For a finite flake of width $W$ and length $L$ we can conclude that the states connect and form chiral states that travel around as indicated in the scheme.

The difference in the color intensity of the edge modes shows the difference in the localization strength. We find three types of states inside the bulk band gap: (i) edge modes outside cones (intervalley states) in the zigzag ribbons; (ii) edge states residing withing one valley (intravalley states) in the zigzag ribbons; (iii) edge states in armchair ribbons which are always intravalley states. Additionally, there are also localized edge states outside the bulk band gap in the zigzag ribbons which have opposite chirality compared to those in the gap.

For a quantitative comparison of the localization we plot the probability amplitudes per atom (unit cell) of states on the $y=0(x=0)$ edge of the zigzag (armchair) nanoribbon in Fig. S6 (analogous results for the other side of the ribbon).

We first focus on the edge states in zigzag ribbons. States (1) and (I) are well localized at the edge. The probability amplitude falls to zero after eight lattice sites. However, state (2) is spread over more than 25 atoms and its probability amplitude is much smaller on the outmost atoms compared to (1) and (I). The reason for this is that (1) and (I) are intervalley states which are separated in $\mathbf{k}$-space from the bulk bands at $\mathrm{K}$ and $\mathrm{K}^{\prime}$. State (2) lives within one valley inside the band gap that is opened by Rashba SOC. So it is spectrally much closer to the bulk bands than intervalley states and its coupling to the bulk can be decreased by increasing the gap, i.e., increasing Rashba SOC. Note that state (1) in case (su) will move towards $\mathrm{K}^{\prime}$ when intrinsic SOC or staggered potential are decreased. For vanishing intrinsic SOC it turns into an intravalley state and the band structure looks similar to the one of case (uu). Consequently, reducing intrinsic SOC declines the localization of state (1).

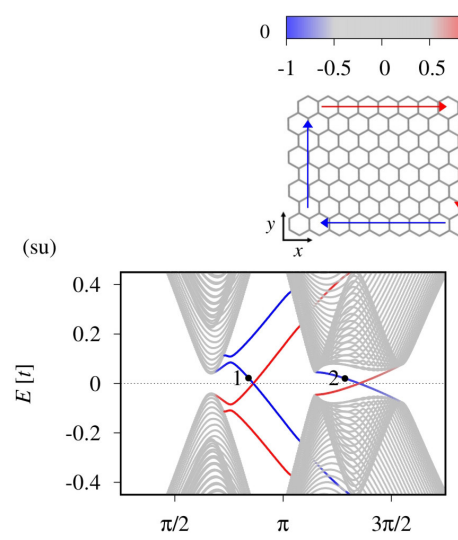

W
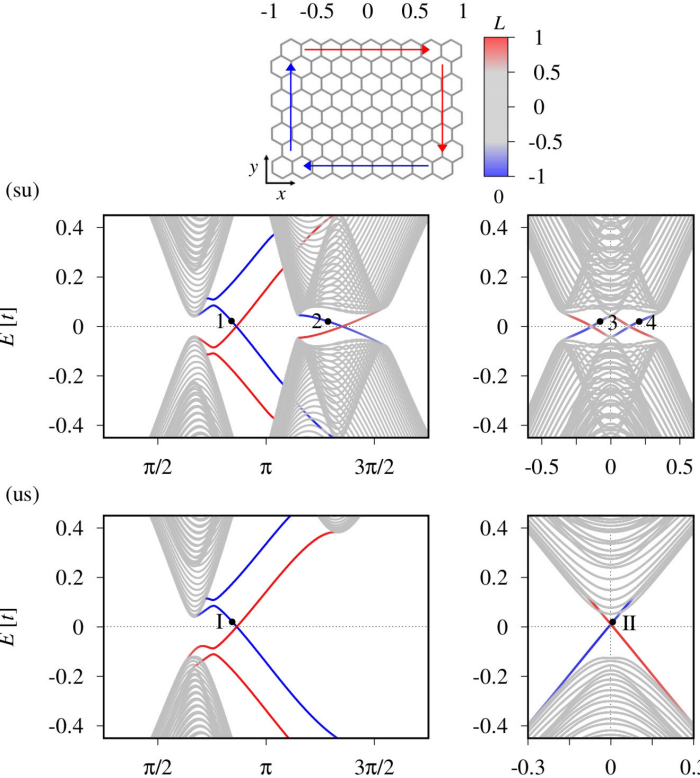

(uu)
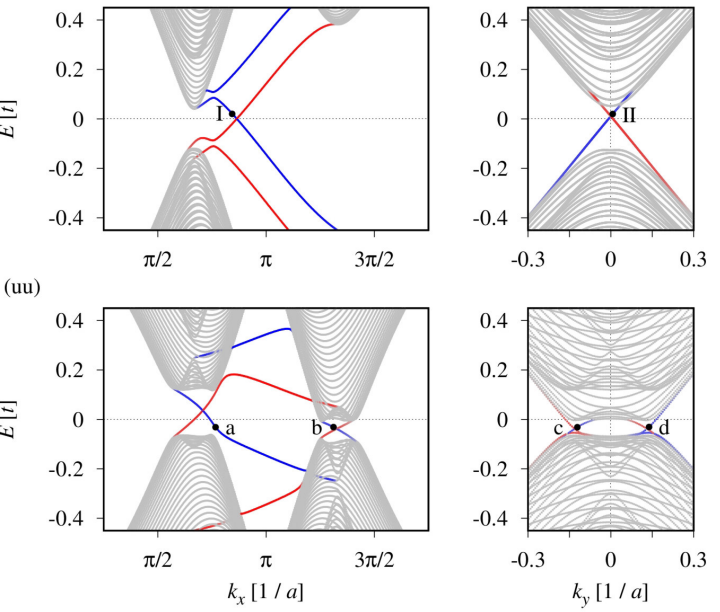

FIG. S5. Calculated band structure of zigzag (left) and armchair (right) nanoribbons with 100 unit cells width. Color indicates localization of states as defined in Eq. (S9). We use $\lambda_{E}^{A}=\left|\lambda_{E}^{B}\right|=0.25 t$ and $\lambda_{I}^{A}=\left|\lambda_{I}^{B}\right|=3 \sqrt{3} \times 0.06 t$ in (su), (us), and $\lambda_{I}^{A}=\left|\lambda_{I}^{B}\right|=0.1 t$ in (uu). Dots specify states presented in Fig. S6.

A decrease of intrinsic SOC in case (us) leads to a transition to a trivial phase (see Fig. 2 in the main part). The QAHE phase in this case and the consequent appearance of intervalley state (I) is caused by intrinsic SOC whereas Rashba SOC is not neccessary and neither sufficient to form a topological phase when exchange coupling is staggered.

From intravalley states (a) and (b) we can see the effect of the size of the band gap in a valley on the localization of intravalley edge states as described above. Staggered potential causes the asymmetry in the size of the band gap in $\mathrm{K}^{\prime}$ and $\mathrm{K}$ and the asymmetric shift of the crossings of the edge states away from zero energy. Increasing Rashba SOC symmetrically enlarges the bulk band gap 

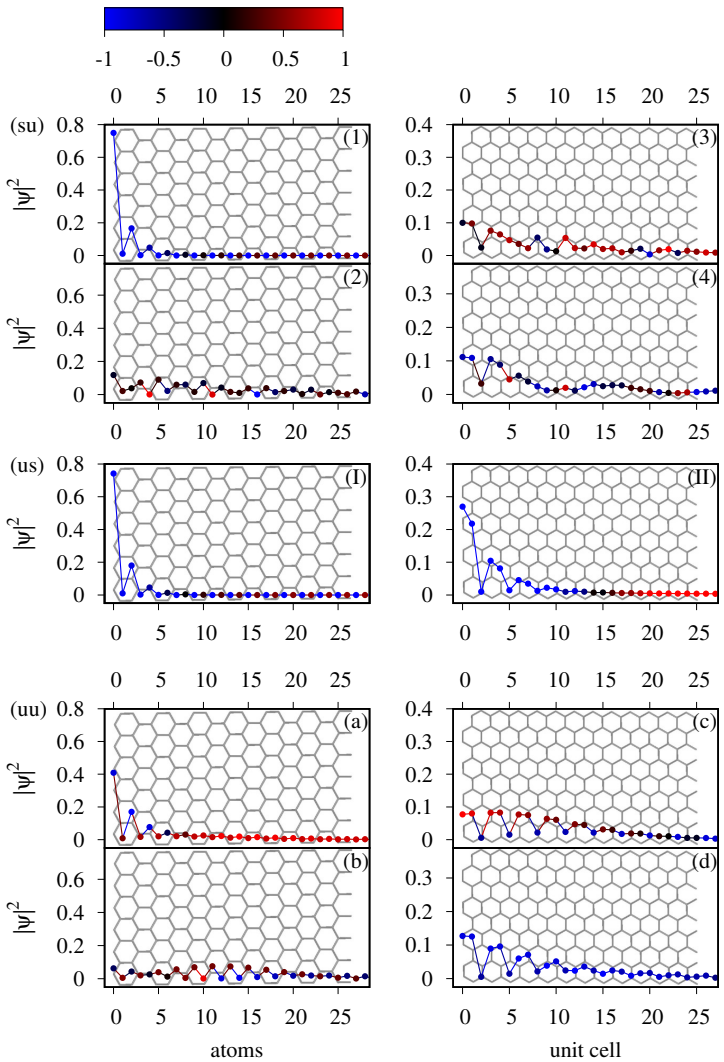

FIG. S6. Probability amplitudes of edge states versus distance from the left edge of zigzag (left panels) and armchair (right panels) nanoribbons with 100 unit cells width. The energy and momentum of the states in the nanoribbon spectrum are marked by dots in Fig. S5. Color indicates $\hat{s}_{z}$ spin density on each site. We use $\lambda_{E}^{A}=\left|\lambda_{E}^{B}\right|=0.25 t$ and $\lambda_{I}^{A}=\left|\lambda_{I}^{B}\right|=$ $3 \sqrt{3} \times 0.06 t$ in (su), (us), and $\lambda_{I}^{A}=\left|\lambda_{I}^{B}\right|=0.1 t$ in (uu).

of the two valleys and strengthens the localization of the states.

Now, we compare the localization behavior of the armchair edge states to what we found for zigzag states. In case $(\mathrm{su})$ the localization for both presented armchair states is similar to the intravalley zigzag state. The reason is the folding of the Brillouin zone such that $\mathrm{K}$ and $\mathrm{K}^{\prime}$ fall on top of each other for armchair terminated ribbons. Thus, states (3) and (4) are both inside the valley gap close to the bulk bands. The size of the gap determines their localization strength. Similar arguments hold for states (c) and (d). For state (II) we also observe a reduced localization compared to its zigzag counterpart because it resides inside the valley. Since the gap is larger than in cases (su) and ( $\mathrm{uu}$ ) state (II) is best localized in

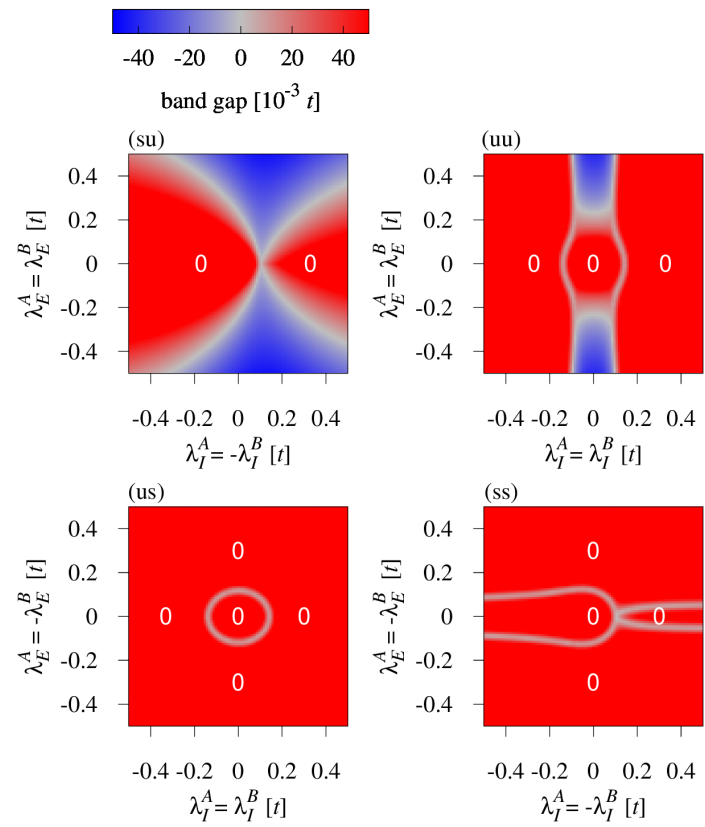

FIG. S7. Global bulk band gap and Chern number (white numbers) for $\Delta=0.1 t$ and $\lambda_{R}=(3 / 2) \times 0.05 t$ varying intrinsic SOC $\lambda_{I}^{A}, \lambda_{I}^{B}$ and exchange splitting $\lambda_{E}^{A}, \lambda_{E}^{B}$ for in-plane magnetization oriented along x-direction, i.e., $\theta=\pi / 2$ and $\phi=0$. Negative band gap indicates transition to metallic phase due to indirect band gap closing from bands at different $\mathbf{k}$ values.

comparison to the other presented armchair modes.

\section{Rotation of magnetization}

In the main paper we state that rotating the orientation of magnetization from $z$ - to $x$-direction turns the system into a trivial insulator or metal. In Figs. S7 and S8 we confirm this by plotting the bulk band gap and Chern numbers for magnetization along $x$ - and $y$-direction, respectively. The phase diagrams look very similar in both cases, there are only small differences in the exact position of some gap closings. For example in case (ss), the grey lines at the right edge of the plot split in Fig. S8 which is not the case in Fig. S7.

Further, we present the evolution of the bulk bands and the zigzag band structures for cases (su), (us), and (ss), when magnetization is gradually rotated from $z$ - to $x$-direction, in Figs. S9 and S10, respectively.

In case (su) we can follow the bulk gap closing and reopening in $\mathrm{K}^{\prime}$ which makes the system trivial. The same happens in case (us). Case (uu) has an indirect gap closing at $k_{y} \neq 0$, which is therefore not visible in 


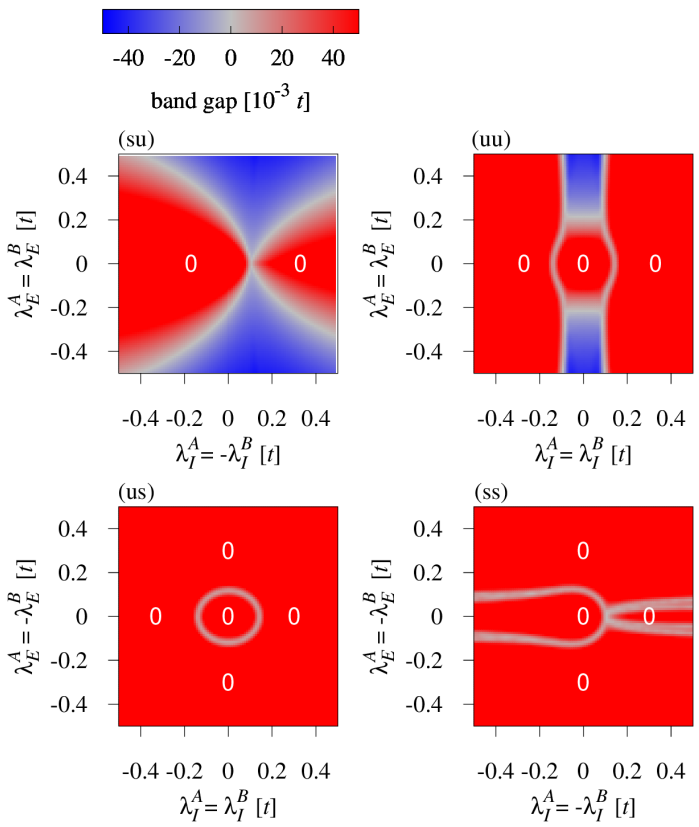

FIG. S8. Global bulk band gap and Chern number (white numbers) for $\Delta=0.1 t$ and $\lambda_{R}=(3 / 2) \times 0.05 t$ varying intrinsic SOC $\lambda_{I}^{A}, \lambda_{I}^{B}$ and exchange splitting $\lambda_{E}^{A}, \lambda_{E}^{B}$ for in-plane magnetization oriented along y-direction, i.e., $\theta=\pi / 2$ and $\phi=\pi / 2$. Negative band gap indicates transition to metallic phase due to indirect band gap closing from bands at different $\mathbf{k}$ values.

Fig. S9(uu), turning the system into a trivial one. In case (ss) the magnetization rotation causes a transition from a metallic to a topologically trivial system.

We can observe the same evolution from the spectra of zigzag nanoribbons in Fig. S10. In case (su) the intervalley states separate and the bulk bands in both cones get close to zero energy. In the zigzag ribbon, there are still states which are localized at the edge but we checked that they are absent in the armchair ribbon in agreement with being a trivial system. The QAHE states in case (us) split and move to higher energy in the spectrum when the corresponding bulk gap closes and reopens, turning the systems into trivial insulators. In case (uu) the edge states merge into the cones when the system enters the trivial insulator phase. The bands in case (ss) follow the transition from metallic to trivial insulator analog to the bulk system.

\section{First-Principles Calculations}

Computational Details and Geometry

The electronic structure calculations and structural relaxation of graphene on $\mathrm{MnPSe}_{3}$ are performed by density functional theory (DFT) [5] with Quantum ESPRESSO [6]. Self-consistent calculations are carried out with the k-point sampling of $30 \times 30 \times 1$ to get converged results for the proximity exchange splittings. We perform open shell calculations that provide the spinpolarized antiferromagnetic ground state of $\mathrm{MnPSe}_{3}$. A Hubbard parameter of $U=5 \mathrm{eV}$ is used for Mn $d$-orbitals, in agreement with recent calculations $[7,8]$. We use an energy cutoff for charge density of 700 Ry and the kinetic energy cutoff for wavefunctions is 65 Ry for the scalar relativistic pseudopotential with the projector augmented wave method [9] with the Perdew-Burke-Ernzerhof exchange correlation functional [10]. For the relaxation of the heterostructures, we add van der Waals corrections $[11,12]$ and use quasi-Newton algorithm based on trust radius procedure. In order to simulate quasi-2D systems, we add a vacuum of $20 \AA$ to avoid interactions between periodic images in our slab geometry. To determine the interlayer distances, the atoms of graphene are allowed to relax only along $z$-direction (vertical to the layers) and the atoms of $\mathrm{MnPSe}_{3}$ are allowed to move in all directions, until every component of each force is reduced below $10^{-3}\left[\mathrm{Ry} / a_{0}\right]$, where $a_{0}$ is the Bohr radius.

The heterostructure of graphene/ $\mathrm{MnPSe}_{3}$ contains a $5 \times 5$ supercell of graphene on a $2 \times 2 \mathrm{MnPSe}_{3}$ supercell. We stretch the lattice constant of graphene [13] from $2.46 \AA$ to $2.48 \AA$ and compress the lattice constant of $\mathrm{MnPSe}_{3}$ by roughly $3 \%$ from $6.387 \AA$ [14] to $6.20 \AA$. The considered heterostructure model has a lattice constant of $12.4 \AA$ and contains 90 atoms in the unit cell. The average interlayer distance is relaxed to $3.36 \AA$.

Low energy model Hamiltonian

From our first principles calculations, we extract the low energy band structure of the proximitized graphene. The following Hamiltonian, derived from symmetry [1517], is able to describe the graphene bands in the vicinity of the Dirac points when only proximity exchange and no $\mathrm{SOC}$ is present

$$
\begin{aligned}
\mathcal{H}_{\mathrm{GR}} & =\mathcal{H}_{0}+\mathcal{H}_{\Delta}+\mathcal{H}_{E}, \\
\mathcal{H}_{0} & =\hbar v_{\mathrm{F}}\left(\kappa k_{x} \sigma_{x}-k_{y} \sigma_{y}\right) s_{0}, \\
\mathcal{H}_{\Delta} & =\Delta \sigma_{z} s_{0}, \\
\mathcal{H}_{E} & =\left(\lambda_{E}^{A} \sigma_{+}-\lambda_{E}^{B} \sigma_{-}\right) s_{z} .
\end{aligned}
$$

We denote $v_{\mathrm{F}}$ as the Fermi velocity and in the linearized Hamiltonian the Cartesian components of the wave vector $k_{x}$ and $k_{y}$ are measured from $\pm \mathrm{K}$. The valley index 

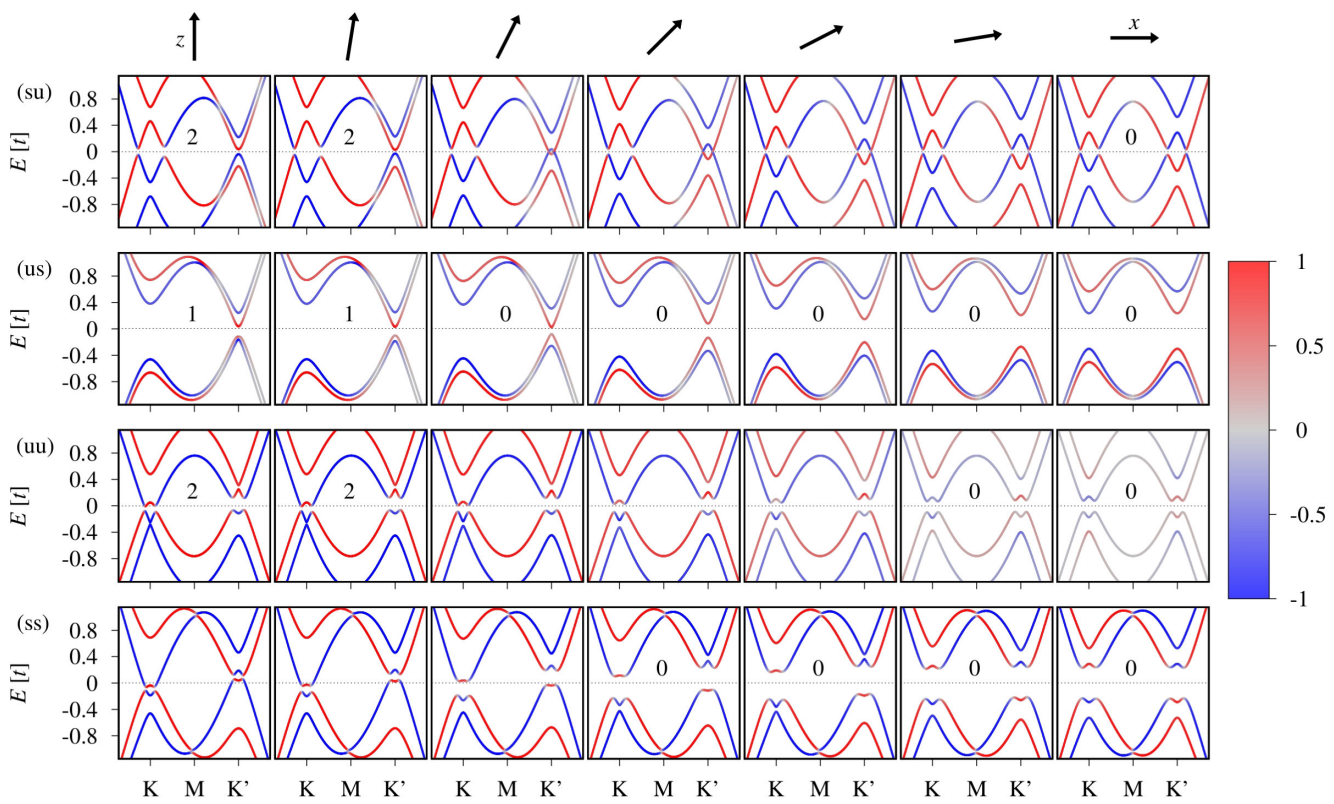

FIG. S9. Calculated bulk band structure for $k_{y}=0$ as magnetization direction evolves from $z$-direction on the left $(\theta=0$, $\phi=0)$ to $x$-direction on the right side $(\theta=\pi / 2, \phi=0)$. Arrows specify direction of magnetization. The numbers are Chern numbers. If no number is specified the system shows a direct or indirect band gap closing [not neccessarily along $k_{y}=0$ as in case (uu)]. Color indicates $\hat{s}_{z}$ spin expectation value and we use $\lambda_{E}^{A}=\left|\lambda_{E}^{B}\right|=0.25 t$ and $\lambda_{I}^{A}=\left|\lambda_{I}^{B}\right|=3 \sqrt{3} \times 0.06 t$ in (su), (us), (ss), and $\lambda_{I}^{A}=\left|\lambda_{I}^{B}\right|=0.1 t$ in (uu).

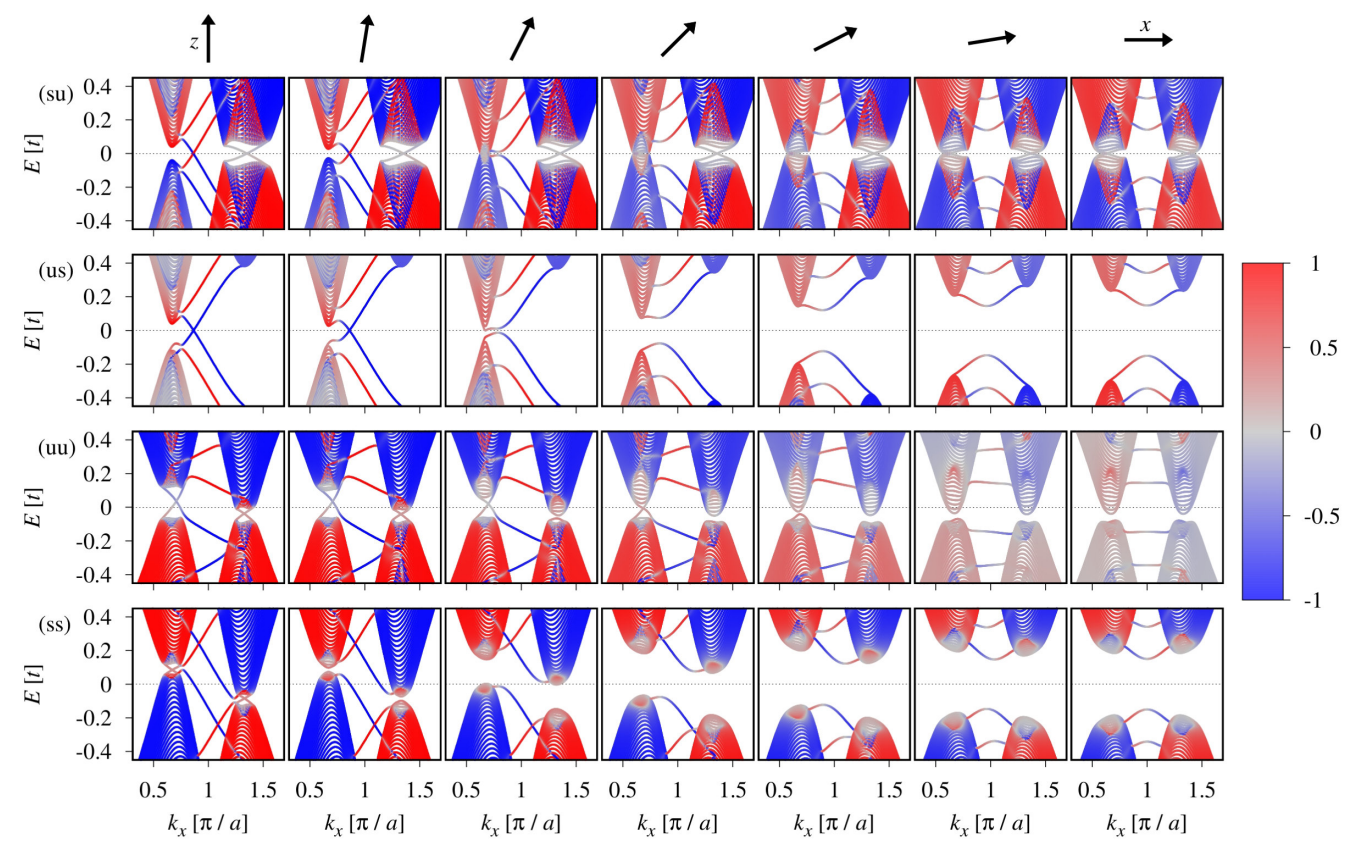

FIG. S10. Calculated electronic structure of zigzag nanoribbons as magnetization direction evolves from $z$-direction $(\theta=0$, $\phi=0)$ to $x$-direction $(\theta=\pi / 2, \phi=0)$. Arrows specify direction of magnetization. Color indicates $\hat{s}_{z}$ spin expectation value and we use $\lambda_{E}^{A}=\left|\lambda_{E}^{B}\right|=0.25 t$ and $\lambda_{I}^{A}=\left|\lambda_{I}^{B}\right|=3 \sqrt{3} \times 0.06 t$ in (su), (us), (ss), and $\lambda_{I}^{A}=\left|\lambda_{I}^{B}\right|=0.1 t$ in (uu). 

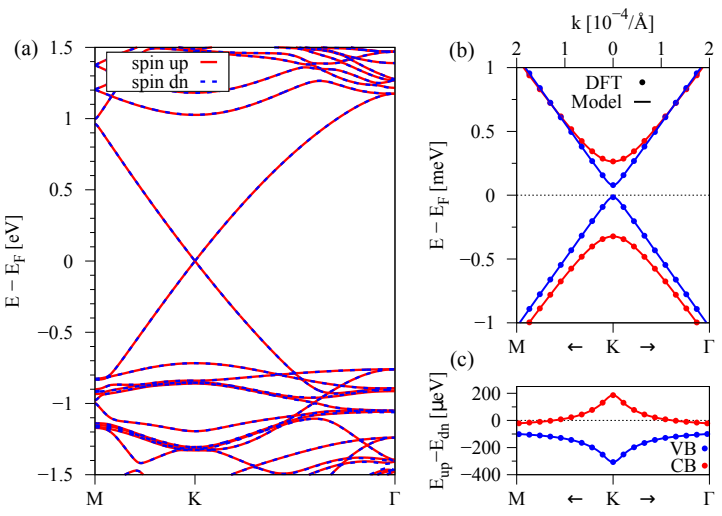

FIG. S11. (a) Calculated electronic band structure of the graphene/ $\mathrm{MnPSe}_{3}$ heterostructure without SOC for an interlayer distance of $3.16 \AA$ A. Bands in red (blue) correspond to spin up (down). (b) Zoom to the calculated low energy bands (symbols) with a fit to the model Hamiltonian $\mathcal{H}_{\mathrm{GR}}$ (solid line). (c) The splitting between spin up and spin down bands $E_{\text {up }}-E_{\text {dn }}$ for the VB and CB.

TABLE S2. Fit parameters of Hamiltonian $\mathcal{H}_{\mathrm{GR}}$ for the graphene/ $\mathrm{MnPSe}_{3}$ heterostructure for different interlayer distances: Fermi velocity $v_{\mathrm{F}}$, staggered potential $\Delta$, proximity exchange parameters $\lambda_{E}^{A}$ and $\lambda_{E}^{B}$, the Dirac point energy $E_{D}$, and the total energy $E_{\text {tot }}$ with respect to the minimum total energy for the relaxed interlayer distance of $3.36 \AA$.

\begin{tabular}{c|ccccccc}
\hline \hline $\begin{array}{c}\text { distance } \\
{[\AA]}\end{array}$ & $\begin{array}{c}v_{\mathrm{F}} \\
{\left[10^{5} \frac{\mathrm{m}}{\mathrm{s}}\right]}\end{array}$ & $\begin{array}{c}\lambda_{E}^{A} \\
\mu \mathrm{eV}]\end{array}$ & $\begin{array}{c}\lambda_{E}^{B} \\
{[\mathrm{eV}]}\end{array}$ & $E_{D}$ & $E_{\mathrm{tot}}$ \\
\hline 3.36 & 8.148 & 49 & 66 & -95 & -420 & 0 \\
3.26 & 8.114 & 76 & 96 & -137 & 32 & 147 \\
3.16 & 8.064 & 123 & 140 & -201 & 1 & 467 \\
\hline \hline
\end{tabular}

is $\kappa= \pm 1$ for $\pm \mathrm{K}$ and the Pauli matrices $s_{i}$, act on spin $(\uparrow, \downarrow)$, and $\sigma_{i}$ on pseudospin $(A, B)$, with $i=0, x, y, z$. For short notation we introduce $\sigma_{ \pm}=\frac{1}{2}\left(\sigma_{z} \pm \sigma_{0}\right)$. The staggered potential is $\Delta$ and the proximity exchange parameters are $\lambda_{E}^{A}$ and $\lambda_{E}^{B}$. As a basis we use the four states $\left|\Psi_{\mathrm{A}}, \uparrow\right\rangle,\left|\Psi_{\mathrm{A}}, \downarrow\right\rangle,\left|\Psi_{\mathrm{B}}, \uparrow\right\rangle$, and $\left|\Psi_{\mathrm{B}}, \downarrow\right\rangle$. Note that the model Hamiltonian is centered around zero energy Fermi level. For the first principles results this is not necessarily the case, as doping can occur. Therefore we introduce another parameter $E_{D}$, which introduces a shift on the global model band structure and we call it the Dirac point energy.

\section{Smaller interlayer distance}

Compared to the band structure in the main text, we want to check if the staggered proximity exchange can be enhanced by decreasing the interlayer distance between graphene and $\mathrm{MnPSe}_{3}$. In Fig. S11 we show the calculated band structure and a zoom to the low energy Dirac bands, when we decrease the relaxed interlayer distance by $0.2 \AA$ to $3.16 \AA$. Indeed, the band splittings $E_{\mathrm{up}}-E_{\mathrm{dn}}$ increase to about $200 \mu \mathrm{eV}$ close to the $\mathrm{K}$ point and are still opposite in sign for $\mathrm{VB}$ and conduction band (CB).

In Table S2 we summarize the fit results of Hamiltonian $\mathcal{H}_{\mathrm{GR}}$ for different interlayer distances. Most interesting is the fact that the two proximity exchange parameters $\lambda_{E}^{A}$ and $\lambda_{E}^{B}$ are opposite in sign and their magnitude can be enhanced by decreasing the distance between graphene and the antiferromagnet $\mathrm{MnPSe}_{3}$.

\section{Intermediate staggered and uniform cases}

For simplicity, we considered so far combinations of exact staggered or uniform intrinsic spin-orbit and exchange coupling. In this section we relax the staggered or uniform condition and discuss intermediate cases. We denote the cases as before (intrinsic SOC - exchange coupling) and indicate the parameter, that is varied, by $\mathrm{x}$, the magnitude of the other parameter is fixed to $\lambda_{I}^{A}=\left|\lambda_{I}^{B}\right|=3 \sqrt{3} \times 0.06 t$ and $\lambda_{E}^{A}=\left|\lambda_{E}^{B}\right|=0.25 t$, respectively.

The phase diagrams are shown in Fig. S12. They demonstrate that the topological phases, which we found in the main paper, do not rely on the condition of exactly staggered or uniform parameters. They allow a significant difference in the absolute value of the parameters for $A$ and $B$ sublattice since the phases that are traversed by the diagonals of the phase space extend over large regions.

This can be seen for the $C= \pm 2$ phase of case (su) in Fig. S12(sx) for the exchange coupling and in Fig. S12(xu) for the intrinsic SOC. The stability of the $C= \pm 1$ phases of case (us) is investigated in Fig. S12(ux) for the exchange coupling. Indeed, $C= \pm 1$ survives when the absolute value of the staggered (opposite sign) exchange coupling parameter on $B$ is about $40 \%$ larger than the one on $A$, as found for the proximity exchange parameters extracted from our DFT calculations for the graphene/ $\mathrm{MnPSe}_{3}$ heterostructure. In Fig. S13 we show the bandstructure of a zigzag and armchair nanoribbon for such a case and find one QAHE state per edge. Since we changed the magnitude of the exchange parameter $\lambda_{E}^{B}$ compared to the results in the main paper, the states that are localized on the $B$ sublattice in the zigzag ribbon are shifted in energy (red states). In the armchair ribbon both edges contain $A$ and $B$ atoms. Consequently, both edge states experience an energy shift. The $C= \pm 1$ 
phases in the (ux) diagram even extend into a region where both exchange coupling parameters have the same sign but different magnitude. Figure S12(xs) shows the strong robustness of the $C= \pm 1$ phase against a deviation from the uniform intrinsic SOC. We find that the phase not only survives a remarkably large difference in the absolute value of the SOC parameters on $A$ and $B$ but even exists for SOC parameters with opposite sign and different absolute values.

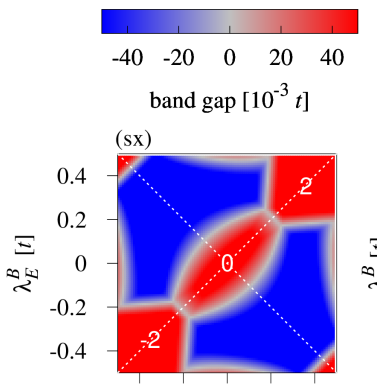

$\begin{array}{cccc}-0.4-0.2 & 0 & 0.2 & 0.4\end{array}$ $\lambda_{E}^{A}[t]$

(ux)

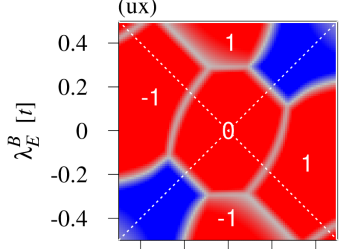

$-0.4-0.2 \quad 0 \quad 0.2 \quad 0.4$ $\lambda_{E}^{A}[t]$

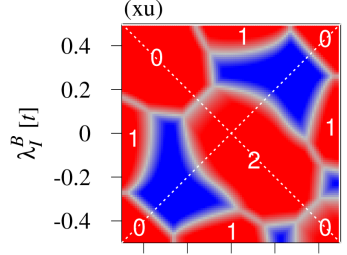

$\begin{array}{cccc}-0.4-0.2 & 0 & 0.2 & 0.4\end{array}$ $\lambda_{I}^{A}[t]$

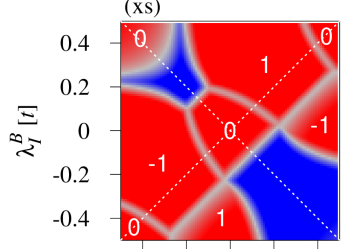

$\begin{array}{cccc}-0.4-0.2 & 0 & 0.2 & 0.4\end{array}$ $\lambda_{I}^{A}[t]$
FIG. S12. Topological phases of magnetic graphene with SOC. Global bulk band gap and Chern number (white numbers) for $\Delta=0.1 t$ and $\lambda_{R}=(3 / 2) \times 0.05 t$ varying exchange splitting $\lambda_{E}^{A}, \lambda_{E}^{B}$ in (sx) and (ux) for fixed staggered/uniform amplitude of intrinsic SOC $\lambda_{I}^{A}=\left|\lambda_{I}^{B}\right|=3 \sqrt{3} \times 0.06 t$; varying intrinsic SOC $\lambda_{I}^{A}, \lambda_{I}^{B}$ in (xu) and (xs) for fixed uniform/staggered amplitude of exchange coupling $\lambda_{E}^{A}=\left|\lambda_{E}^{B}\right|=0.25 t$. The dotted white lines mark the diagonals of the phase diagrams where the considered parameters are exactly uniform or staggered. Magnetization direction is oriented along $\hat{\mathbf{m}}=(0,0,1)$. Negative band gap indicates transition to metallic system due to indirect band gap closing from bands at different $\mathbf{k}$ values.

Furthermore, the phase space becomes richer, when we allow for different absolute values of intrinsic spin-orbit and exchange coupling on $A$ and $B$ sublattice, introducing additional topologically nontrivial phases.

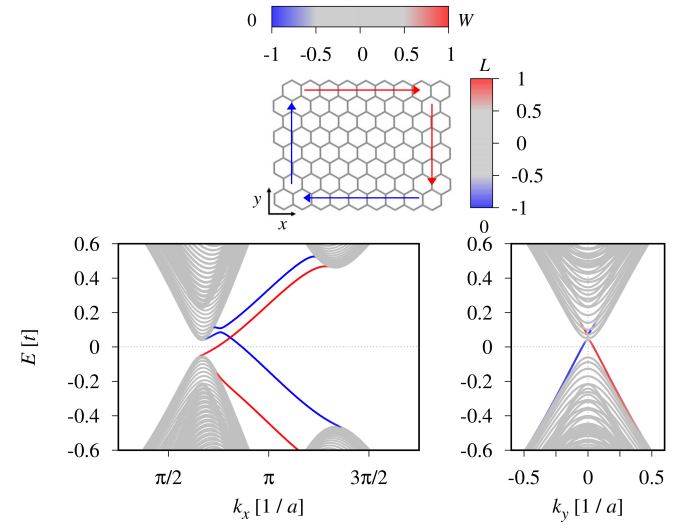

FIG. S13. Calculated band structure of zigzag (left) and armchair (right) nanoribbon with 100 unit cells width for case (ux). We use $\lambda_{I}^{A}=\left|\lambda_{I}^{B}\right|=3 \sqrt{3} \times 0.06 t$ and $\lambda_{E}^{A}=0.25 t$, $\lambda_{E}^{B}=-0.36 t$. Color indicates localization of states as defined in Eq. (S9).

\section{Robustness with respect to uniform disorder}

Both cases (us) and (su) host topologically protected states indicated by the Chern number of the bulk system, which are well recognizable in the band structure of zigzag ribbons. In order to give more qualitative insight into how well these states are protected, we need to give an energy scale. Here, we investigate the behavior of low-energy states of finite flakes, which are mostly zigzag-edge terminated, under uniform onsite disorder. We add random onsite disorder on each lattice site, being the same for each spin, i.e. preserving time-reversal symmetry. The onsite disorder is randomly drawn from a uniform distribution within the interval $\left[-V_{d}, V_{d}\right]$ with the disorder strength $V_{d}$. This type of disorder could be present when graphene is placed onto a rough substrate, which induces charge puddles, local variations in the potentials (although this disorder type has a longrange correlation).

In Fig. S14(us) and (su) we show typical low-energy states of such flakes in the presence of disorder, where the disorder strength is indicated in the figure. This gives a qualitative picture of the energy scale of the disorder strength up to which the states are still robust against the disorder. For case (us) the states start to differ from the expected spin distribution at about $0.5 t$, where they can scatter into different spin polarized states. This energy is on the order of twice the exchange energy or the intrinsic spin-orbit coupling, which are responsible for gap opening in the system.

The case (su) shows well known [18] local spinpolarized pseudohelical states. They are in accordance with the findings of the zigzag band structure study in the 
(us)

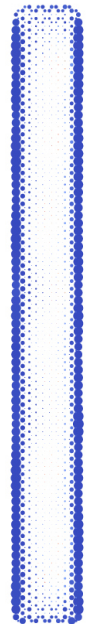

$0.1 t$

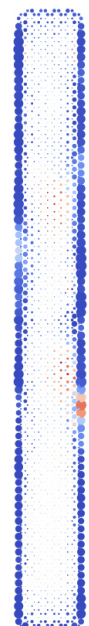

$0.5 t$

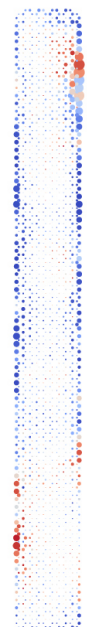

$1.0 t$ (su)

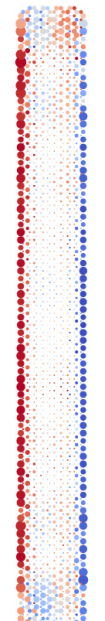

$0.1 t$

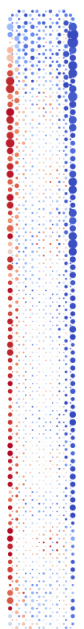

$0.3 t$

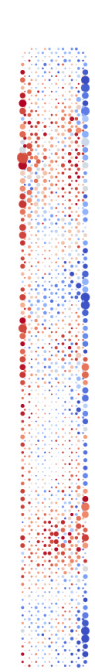

$0.5 t$

FIG. S14. Uniform disorder study of quantum anomalous Hall effects in graphene for low-energy states in finite flakes. Flakes considered are of the size $80 \times 10$ graphene unit cells. The left part (us) shows the case of uniform intrinsic SOC and staggered exchange, with different disorder strengths $V_{d}$. The right part $(\mathrm{su})$ displays low-energy states for staggered intrinsic SOC and uniform exchange. Dot positions indicate the lattice, their size denotes the weight of the state on that position and color codes the local spin- $z$ expectation value (up in red and down in blue).

main text. The only difference is that the spinless states (from the zigzag band calculation), centered within one valley, are absent due to finite size effects (this is known behavior, very analogous to the exchange-less case [18]). It is also interesting in that case to study the behavior with respect to random onsite disorder. These local spinpolarized states behave very similar to their exchange-less counterpart, being robust up to a disorder strength of about $0.3 t$.

In conclusion, states from the (us) and (su) cases are robust against random onsite disorder, moreover they should withstand magnetic disorder, as time-reversal

symmetry is already broken here by the exchange parts. The quantitative behavior with respect to disorder calls for a more detailed study.

* Emails to: petra.hoegl@physik.uni-regensburg.de

1] D. J. Thouless, M. Kohmoto, M. P. Nightingale, and M. den Nijs, Phys. Rev. Lett. 49, 405 (1982).

[2] S. Ryu, A. P. Schnyder, A. Furusaki, and A. W. W. Ludwig, New J. Phys. 12 (2010).

[3] Z. Qiao, H. Jiang, X. Li, Y. Yao, and Q. Niu, Phys. Rev. B 85, 115439 (2012).

[4] Y. Ren, Z. Qiao, and Q. Niu, Rep. Prog. Phys. 79, 066501 (2016).

[5] P. Hohenberg and W. Kohn, Phys. Rev. 136, B864 (1964).

[6] P. Giannozzi, S. Baroni, N. Bonini, M. Calandra, R. Car, C. Cavazzoni, D. Ceresoli, G. L. Chiarotti, M. Cococcioni, I. Dabo, A. D. Corso, S. Fabris, G. Fratesi, S. de Gironcoli, R. Gebauer, U. Gerstmann, C. Gougoussis, A. Kokalj, M. Lazzeri, L. Martin-Samos, N. Marzari, F. Mauri, R. Mazzarello, S. Paolini, A. Pasquarello, L. Paulatto, C. Sbraccia, S. Scandolo, G. Sclauzero, A. P. Seitsonen, A. Smogunov, P. Umari, and R. M. Wentzcovitch, J. Phys.: Condens. Mat. 21, 395502 (2009).

[7] W. Mi, X. Wang, J. Zou, Y. Song, and Q. Pei, Sci. Rep. 7, 1 (2017)

[8] Q. Pei, X. Wang, J. Zou, and W. Mi, Nanotechnology 29, 214001 (2018)

[9] G. Kresse and D. Joubert, Phys. Rev. B 59, 1758 (1999).

[10] J. P. Perdew, K. Burke, and M. Ernzerhof, Phys. Rev. Lett. 77, 3865 (1996).

[11] S. Grimme, J. Comput. Chem. 27, 1787 (2006).

[12] V. Barone, M. Casarin, D. Forrer, M. Pavone, M. Sambi, and A. Vittadini, J. Comput. Chem. 30, 934 (2009).

[13] Y. Baskin and L. Meyer, Phys. Rev. 100, 544 (1955).

[14] A. Wiedenmann, J. Rossat-Mignod, A. Louisy, R. Brec, and J. Rouxel, Solid State Commun. 40, 1067 (1981).

[15] D. Kochan, S. Irmer, and J. Fabian, Phys. Rev. B 95, 165415 (2017).

[16] V. T. Phong, N. R. Walet, and F. Guinea, 2D Mater. 5, 014004 (2017).

[17] K. Zollner, M. Gmitra, T. Frank, and J. Fabian, Phys. Rev. B 94, 155441 (2016).

[18] T. Frank, P. Högl, M. Gmitra, D. Kochan, and J. Fabian, Phys. Rev. Lett. 120, 156402 (2018). 